\title{
Nonadiabatic dynamics with spin-flip versus linear-response time-dependent density functional theory: A case study for the protonated Schiff base $\mathrm{C}_{5} \mathrm{H}_{6} \mathrm{NH}_{2}^{+}$
}

\author{
Xing Zhang \\ Department of Chemistry and Chemical Engineering, \\ California Institute of Technology, Pasadena, CA 91125 \\ John M. Herbert* \\ Department of Chemistry and Biochemistry, The Ohio State University, Columbus, OH 43210
}

(Dated: July 8, 2021)

\begin{abstract}
Nonadiabatic trajectory surface hopping simulations are reported for trans $-\mathrm{C}_{5} \mathrm{H}_{6} \mathrm{NH}_{2}^{+}$, a model of the rhodopsin chromophore, using the augmented fewest-switches algorithm. Electronic structure calculations were performed using time-dependent density functional theory (TDDFT) in both its conventional linear-response (LR) and its spin-flip (SF) formulations. In the SF-TDDFT case, spin contamination in the low-lying singlet states is removed by projecting out the lowest triplet component during iterative solution of the TDDFT eigenvalue problem. The results show that SF-TDDFT is able to correctly describe the photoisomerization of trans $-\mathrm{C}_{5} \mathrm{H}_{6} \mathrm{NH}_{2}^{+}$, with favorable comparison to previous studies using multireference electronic structure methods. In contrast, conventional LRTDDFT affords qualitatively different photodynamics due to an incorrect excited-state potential surface near the Franck-Condon region. In addition, the photochemistry (involving pre-twisting of the central double bond) appears to be different for SF- and LR-TDDFT, which may be a consequence of different conical intersection topographies afforded by these two methods. The present results contrast with previous surface-hopping studies suggesting that the LR-TDDFT method's incorrect topology around $\mathrm{S}_{1} / \mathrm{S}_{0}$ conical intersections is immaterial to the photodynamics.
\end{abstract}

\section{INTRODUCTION}

Time-dependent density functional theory (TDDFT), in its conventional linear-response (LR) formulation, ${ }^{1-3}$ fails to describe the topology of any conical intersection (CX) that involves the reference state (typically the ground state), for which the branching space is onedimensional rather than two-dimensional. ${ }^{4,5}$ Depending upon one's point of view, this behavior arises either due to a lack of double excitations in the ansatz for the TDDFT "wave function", ${ }^{4}$ or else simply due to an imbalance in the nature of how LR-TDDFT describes the variational ground state versus the response (excited) states, leading to problems in regions of configuration space where the ground state becomes degenerate. A simple example is $\mathrm{H}_{3}$ radical, which is a Jahn-Teller system in its $D_{3 h}$ geometry and for which LR-TDDFT predicts highly distorted potential energy surfaces that fail to reproduce the ground-state degeneracy. ${ }^{6}$

In contrast, CXs between two excited states are topologically correct in LR-TDDFT, which can be understood by considering the configuration interaction singles (CIS) method as a special case and examining its wave function ansatz,

$$
\left|\Psi_{\mathrm{CIS}}\right\rangle=c_{0}\left|\Phi_{0}\right\rangle+\sum_{i a} c_{i a}\left|\Phi_{i a}\right\rangle,
$$

where $\left|\Phi_{i a}\right\rangle$ represents a singly-excited Slater determinant formed from ground-state molecular orbitals (MOs).

*herbert@chemistry.ohio-state.edu
The coefficients $c_{i a}$ are obtained by diagonalizing the orbital Hessian ("A matrix"), ${ }^{3}$ and as such the CIS excited states are variational with respect to one another. However, the ground-state coefficient $c_{0}$ is determined (if needed) by normalization rather than by any variational calculation that involves the excited states.

The "spin-flip" (SF) approach to TDDFT ${ }^{7,8}$ has been used as a means to fix (or at least circumvent) the topology problem. ${ }^{4-6}$ SF-TDDFT starts from a ground-state calculation with higher spin multiplicity as compared to the states of interest, e.g., a triplet state if targeting singlet photochemistry, or a quartet state for the aforementioned doublet $\mathrm{H}_{3}$ radical. This high-spin state is used as a reference for a LR-TDDFT calculation that also involves a one-electron $\alpha \rightarrow \beta$ spin flip, thus affording states with the target multiplicity, at least in favorable cases. (As discussed later in this work, SF-TDDFT is often beset by significant spin contamination. ${ }^{5,9-12}$ ) The SF-TDDFT approach cures the topology problem around a CX by introducing a limited set of double excitations that couple ground and excited states, or (from a different point of view) because all of the states having the target multiplicity are generated by response theory and are thus described in a balanced fashion. Challenging cases such as $\mathrm{H}_{3}$ in $D_{3 h}$ symmetry are described correctly by SF-TDDFT. ${ }^{6}$

Despite its topology problems, however, conventional LR-TDDFT remains a widely-used approach in the context of trajectory surface hopping simulations of nonadiabatic molecular dynamics (NMD). ${ }^{13-20}$ Provided that trajectories are halted before they can undergo internal conversion (IC) to the ground state, problems associated with incorrect topology may be avoided, but this con- 


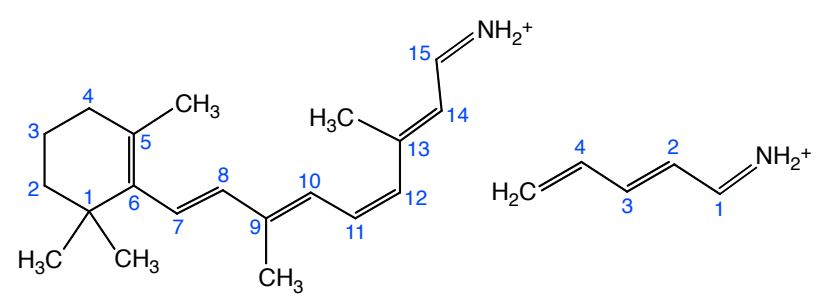

(a) PSB11

(b) PSB3

FIG. 1: Chemical structures of (a) 11-cis-retinal protonated Schiff base (PSB11), and (b) the truncated model, transPSB3.

straint is rather limiting insofar as the timescale for IC is one of the most basic questions that one might wish to answer using NMD simulations. As a counterpoint, there might be reason for optimism given that most nonadiabatic events likely occur near conical seams, where the energy gap is small, as opposed to occurring at a conical seam, where the energy gap is zero but which occupies a vanishingly small volume in the configuration space of a polyatomic molecule. A recent side-by-side comparison of NMD simulations using LR- and SF-TDDFT, ${ }^{21}$ for cis $\rightarrow$ trans photoisomerization azobenzene, seems to corroborate the optimistic viewpoint. That study found very little difference between the two approaches when it comes to predicting either the IC timescale or the branching ratio of cis versus trans photoproducts. It is worth noting, however, that the excited-state trajectories in that study are significantly more oscillatory when LR-TDDFT is used, which may reflect warping of the potential energy surfaces in the vicinity of the $S_{1} / S_{0}$ intersection. In addition, 41 of the 300 LR-TDDFT trajectories had to be discarded due to convergence failure, ${ }^{21}$ whereas none of the SF-TDDFT trajectories suffered this problem. These observations are consistent with the problematic description of CXs involving the ground state in LR-TDDFT. ${ }^{6}$

In the present work, we contribute to this ongoing discussion by comparing NMD simulations using both LR- and SF-TDDFT, for a well-studied photochemical problem: photoisomerization of the protonated Schiff base $\mathrm{C}_{5} \mathrm{H}_{6} \mathrm{NH}_{2}^{+}$. This molecule has been suggested ${ }^{22,23}$ as a minimalist model of the rhodopsin chromophore, ${ }^{24}$ namely, 11-cis-retinal protonated Schiff base (PSB11). The full chromophore is depicted in Fig. 1(a) and the truncated model, trans- $\mathrm{C}_{5} \mathrm{H}_{6} \mathrm{NH}_{2}^{+}$(PSB3), is shown in Fig. 1(b). PSB3 has become something of a benchmark case for excited-state electronic structure theory, ${ }^{25}$ and is used here to probe difference between trajectory surfacehopping results at the LR- and SF-TDDFT levels of theory.

\section{THEORY}

\section{A. Nonadiabatic couplings}

We briefly review the formalism of nonadiabatic couplings in both CIS and LR-TDDFT in order to demonstrate the origin of the topology problem for CXs that involve the reference state. For LR-TDDFT, we use a "pseudo-wave function" formalism in which one treats the Kohn-Sham determinant as a wave function and derivative couplings are obtained from analytic gradient theory, ${ }^{6,26,27}$ in analogy to the way in which nonadiabatic couplings are derived for the CIS method. This approach sidesteps certain divergences associated with the use of quadratic response theory to obtain derivative couplings. ${ }^{28-31}$

In CIS, the electronic wavefunction is expressed as a linear combination of singly-excited Slater determinants $\left|\Phi_{i a}\right\rangle$, as in Eq. (1). The coefficients $c_{0}$ and $\left\{c_{i a}\right\}$ in Eq. (1) are determined by solving the Schrödinger equation $\hat{H}|\Psi\rangle=E|\Psi\rangle$ after projecting the Hamiltonian onto the single excitation space:

$$
\hat{H}=\left|\Phi_{0}\right\rangle E_{0}\left\langle\Phi_{0}\left|+\sum_{i j a b}\right| \Phi_{i a}\right\rangle\left\langle\Phi_{i a}|\hat{H}| \Phi_{j b}\right\rangle\left\langle\Phi_{j b}\right| .
$$

Here, $E_{0}$ is the Hartree-Fock (HF) energy. We use $i, j, \ldots$ to indicate occupied MOs and $a, b, \ldots$ for virtual MOs, as determined by the HF reference state.

Now suppose that two electronic states $\left|\Psi_{I}\right\rangle$ and $\left|\Psi_{J}\right\rangle$ become degenerate at a geometry $\mathbf{R}_{\mathrm{CX}}$. It is possible to define the so-called "crude" adiabatic basis, ${ }^{32}$

$$
\left\{\left|\Psi_{I}\left(\mathbf{R}_{\mathrm{CX}}\right)\right\rangle,\left|\Psi_{J}\left(\mathbf{R}_{\mathrm{CX}}\right)\right\rangle,\left|\widetilde{\Psi}_{K}\left(\mathbf{R}_{\mathrm{CX}}\right)\right\rangle\right\}
$$

in which $\left|\widetilde{\Psi}_{K}\left(\mathbf{R}_{\mathrm{CX}}\right)\right\rangle$ is orthogonal to the two degenerate states. If we further project the Hamiltonian onto the two-dimensional subspace spanned by $\left|\Psi_{I}\left(\mathbf{R}_{\mathrm{CX}}\right)\right\rangle$ and $\left|\Psi_{J}\left(\mathbf{R}_{\mathrm{CX}}\right)\right\rangle$, at an arbitrary geometry $\mathbf{R}$, then the energies of the two intersecting states can be obtained through first order ${ }^{32}$ by diagonalizing the $2 \times 2$ Hamiltonian

$$
\mathbf{H}(\mathbf{R})=\left(\begin{array}{cc}
H_{I I}(\mathbf{R}) & H_{I J}(\mathbf{R}) \\
H_{J I}(\mathbf{R}) & H_{J J}(\mathbf{R})
\end{array}\right)
$$

where

$$
H_{I J}(\mathbf{R})=\left\langle\Psi_{I}\left(\mathbf{R}_{\mathrm{CX}}\right)|\hat{H}(\mathbf{R})| \Psi_{J}\left(\mathbf{R}_{\mathrm{CX}}\right)\right\rangle .
$$

This $2 \times 2$ Hamiltonian becomes diagonal, with degenerate eigenvalues, at the point $\mathbf{R}=\mathbf{R}_{\mathrm{CX}}$ :

$$
\mathbf{H}\left(\mathbf{R}_{\mathrm{CX}}\right)=\left(\begin{array}{cc}
E_{I} & 0 \\
0 & E_{I}
\end{array}\right) .
$$

One can also expand $\mathbf{H}(\mathbf{R})$ around $\mathbf{R}_{\mathrm{CX}}$ to the first order as

$$
\mathbf{H}(\mathbf{R})=\mathbf{H}\left(\mathbf{R}_{\mathrm{CX}}\right)+\hat{\nabla}_{\mathbf{R}} \mathbf{H}\left(\mathbf{R}_{\mathrm{CX}}\right) \cdot \delta \mathbf{R}
$$


where $\delta \mathbf{R}=\mathbf{R}-\mathbf{R}_{\mathrm{CX}}$. To locate another crossing point $\mathbf{R}_{\mathrm{CX}}^{\prime}$ in the vicinity of $\mathbf{R}_{\mathrm{CX}}$, one may require that

$$
\begin{aligned}
& H_{I I}\left(\mathbf{R}_{\mathrm{CX}}^{\prime}\right)=H_{J J}\left(\mathbf{R}_{\mathrm{CX}}^{\prime}\right) \\
& H_{I J}\left(\mathbf{R}_{\mathrm{CX}}^{\prime}\right)=0 .
\end{aligned}
$$

Using Eq. (6), the two conditions in Eq. (7) can be rewritten as

$$
\begin{aligned}
& \mathbf{g}_{I J}\left(\mathbf{R}_{\mathrm{CX}}\right) \cdot \delta \mathbf{R}=0 \\
& \mathbf{h}_{I J}\left(\mathbf{R}_{\mathrm{CX}}\right) \cdot \delta \mathbf{R}=0
\end{aligned}
$$

by introducing the gradient difference vector $\mathbf{g}_{I J}$ and the nonadiabatic coupling vector $\mathbf{h}_{I J}$. The former is defined as

$$
\mathbf{g}_{I J}(\mathbf{R})=\hat{\nabla}_{\mathbf{R}}\left[E_{I}(\mathbf{R})-E_{J}(\mathbf{R})\right]
$$

where

$$
\hat{\nabla}_{\mathbf{R}} E_{I}(\mathbf{R})=\left\langle\Psi_{I}(\mathbf{R})\left|\hat{\nabla}_{\mathbf{R}} \hat{H}(\mathbf{R})\right| \Psi_{I}(\mathbf{R})\right\rangle .
$$

The nonadiabatic coupling is defined as

$$
\mathbf{h}_{I J}(\mathbf{R})=\left\langle\Psi_{I}(\mathbf{R})\left|\hat{\nabla}_{\mathbf{R}} \hat{H}(\mathbf{R})\right| \Psi_{J}(\mathbf{R})\right\rangle .
$$

The vectors $\mathbf{g}_{I J}$ and $\mathbf{h}_{I J}$ span a two-dimensional branching plane at the $\mathrm{CX},{ }^{33}$ and the degeneracy between the two intersecting states is preserved through first order $(\delta \mathbf{R})$, provided that $\delta \mathbf{R}$ is restricted to the space orthogonal to the branching plane, i.e., to the seam space. ${ }^{32}$

If the CX involves the reference state (meaning the HF ground state, for conventional CIS), then the derivation presented in Appendix A shows that the nonadiabatic coupling vector can be written

$$
\mathbf{h}_{0 I}^{\mathrm{CIS}}=-\left(E_{I}-E_{0}\right) \sum_{i a}\left\langle a \mid i^{[\mathbf{R}]}\right\rangle c_{i a}^{I},
$$

where the parameters $c_{i a}^{I}$ are the CIS coefficients for excited state $\left|\Psi_{I}^{\mathrm{CIS}}\right\rangle$ and the superscript $[\mathbf{R}]$ denotes a nuclear derivative. The quantity $\left\langle a \mid i^{[\mathbf{R}]}\right\rangle$ represents the overlap between an unperturbed virtual orbital $|a\rangle$ and a perturbed occupied orbital, $\left|i^{[\mathbf{R}]}\right\rangle$. It is clear from Eq. (12) that the nonadiabatic coupling $\mathbf{h}_{0 I}$ vanishes at any crossing point between the HF ground state and a CIS excited state $\left(E_{0}=E_{I}\right)$, hence the degeneracy is lifted in the direction of $\mathbf{g}_{0 I}$ only and the branching space is one-dimensional. So long as the excitation energy gap $E_{I}-E_{0}$ is not exactly zero, however, the coupling $\mathbf{h}_{0 I}$ does not generally vanish. This makes the topology of the potential surface rather complicated in regions close to crossing points $\mathbf{R}_{\mathrm{CX}}$; see Appendix B.

It should be noted that the nonadiabatic coupling defined in Eq. (11) is slightly different from the one defined by Yarkony, ${ }^{32}$ the latter of which has the following form:

$$
\mathbf{h}_{I J}(\mathbf{R})=\mathbf{c}^{I}(\mathbf{R})^{\dagger} \hat{\nabla}_{\mathbf{R}} \mathbf{H}(\mathbf{R}) \mathbf{c}^{J}(\mathbf{R}) .
$$

The difference comes from the fact that the crude adiabatic basis in Ref. 32 is defined in such a way that only the CI coefficients are fixed, whereas in our definition both the CI coefficients and the Slater determinants are fixed. As a result of Brillouin's theorem, the definition in Eq. (13) makes the nonadiabatic couplings vanish between the HF ground state and the CIS states at any geometric coordinates $\mathbf{R}$. This choice is not convenient for examining the topology of potential energy surfaces at approximate crossing points; see Appendix B. When applied to compute nonadiabatic couplings between two CIS excited states, the use of Eq. (11) versus Eq. (13) affords couplings that differ by so-called electronic translation factors, ${ }^{34}$ equivalent to non-HellmannFeynman terms in the analytic gradient expression for the coupling. ${ }^{6}$

As shown in Appendix A, the nonadiabatic coupling between the Kohn-Sham DFT ground state and LRTDDFT excited states can be defined as

$$
\mathbf{h}_{0 I}^{\mathrm{DFT}}=-\left(E_{I}-E_{0}\right) \sum_{i a}\left\langle a \mid i^{[\mathbf{R}]}\right\rangle\left(x_{i a}^{I}-y_{i a}^{I}\right),
$$

where $x_{i a}^{I}$ and $y_{i a}^{I}$ are the usual LR excitation $\left(x_{i a}^{I}\right)$ and de-excitation $\left(y_{i a}^{I}\right)$ amplitudes for the $I$ th excited state. $^{2,3}$ If the Tamm-Dancoff approximation (TDA) is invoked, ${ }^{3}$ corresponding to neglecting the de-excitation amplitudes, then $x_{i a}^{I}$ coincides with $c_{i a}^{I}$ in Eq. (12). Importantly, however, while the expressions for $\mathbf{h}_{0 I}^{\mathrm{CIS}}$ and $\mathbf{h}_{0 I}^{\text {DFT }}$ are formally very similar, the latter might be nonzero even at a crossing point. This is more clear in the complete-basis limit, where $\mathbf{h}_{0 I}^{\mathrm{DFT}}$ reduces to the Chernyak-Mukamel expression, ${ }^{35-37}$

$$
\mathbf{h}_{0 I}^{\mathrm{DFT}}(\mathbf{R})=\int \rho_{0 I}(\mathbf{r} ; \mathbf{R}) \hat{\nabla}_{\mathbf{R}} v_{\mathrm{ne}}(\mathbf{r} ; \mathbf{R}) d \mathbf{r} .
$$

The quantity $\rho_{0 I}(\mathbf{r} ; \mathbf{R})$ is the transition density, which is a parametric function of the nuclear coordinates $\mathbf{R}$, and $v_{\text {ne }}(\mathbf{r} ; \mathbf{R})$ is the nuclear-electron Coulomb (or "external") potential. Upon invoking the TDA, one finds that $\mathbf{h}_{0 I}^{\mathrm{DFT}}$ in Eq. (14) vanishes whenever $E_{I}=E_{0}$, just like the situation for CIS. (See Appendix A.)

In summary, the nonadiabatic coupling at an approximate conical intersection that involves the reference state does not vanish in general for either CIS or LR-TDDFT, meaning that the branching space is not solely determined by the vector $\mathrm{g}_{0 I}$, as shown in detail in Appendix B along with a numerical example. Nevertheless, the magnitude of these couplings remains small, which is a direct consequence of the absence of proper interactions between the reference state and the response states. The practical consequence is incorrect local topology and large curvature of potential energy surfaces in the vicinity of CXs that involve the reference state. Presumably, this should have consequences for NMD simulations.

\section{B. SF-TDDFT}

Unlike LR-TDDFT, the SF-TDDFT approach treats electron correlation on a consistent footing for both the 


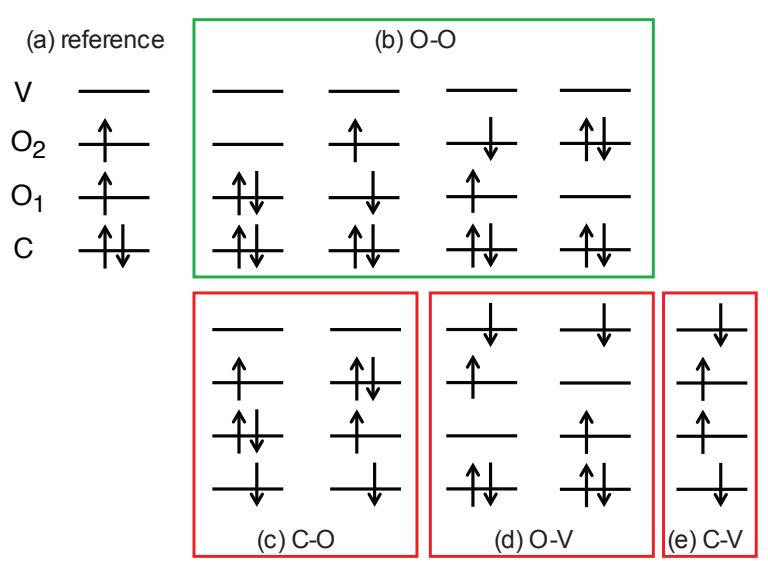

FIG. 2: SF-TDDFT excitation manifold for a four-electron, four-orbital model with a high-spin triplet reference configuration that is shown in (a). The excitation subspace in (b) is spin-complete but those in (c)-(e) are not. Reprinted from Ref. 12; copyright 2015 American Institute of Physics.

ground and excited states of the target multiplicity, and in particular affords correct topology in the vicinity of any CX, including ones that involve the ground state of the target multiplicity. ${ }^{5,6}$ However, the usual SF-TDDFT approach contains only single spin flips, which often leads to significant spin contamination especially as one moves away from the Franck-Condon region of the excited state and starts to explore bond-breaking geometries. The more pervasive spin contamination in SFTDDFT as compared to (spin-conserving) LR-TDDFT can be understood using the simple model that is shown in Fig. 2. ${ }^{5,12}$ Here, a high-spin triplet configuration is used as the reference determinant in order to target states of singlet multiplicity via single excitations combined with a single $\alpha \rightarrow \beta$ spin flip. The figure depicts the SF-TDDFT excitation manifold that is generated in this manner.

Amongst the Slater determinants contained in this manifold, only the four that are depicted in Fig. 2(b) are able to form spin-pure states, i.e., eigenfunctions of $\hat{S}^{2}$, and these can be combined to form three singlet states and one triplet. These states include both the nominal $\mathrm{S}_{0}$ configuration as well as the two determinants necessary to form the properly spin-adapted open-shell $S_{1}$ configuration, corresponding to excitation between frontier MOs. Also present in the "o-o" space of Fig. 2(b) is a double excitation that couples $S_{0}$ and $S_{1}$ and is needed in order to obtain proper topology at the $\mathrm{S}_{1} / \mathrm{S}_{0} \mathrm{CX}{ }^{4}$ The determinants shown in Fig. 2(c)-(e) are also part of the SF-TDDFT excitation manifold, however, and each of these is missing one or more of its "spin complements", meaning the determinants that are necessary to form a $\hat{S}^{2}$ eigenstate with $S=0$. The missing determinants cannot be formed by any single excitation and single spin flip from the high-spin triplet reference state in Fig. 2(a). Any SF-TDDFT state that has a significant contribution from one or more of the determinants in Fig. 2(c)-(e) will exhibit significant spin contamination.

The spin contamination problem becomes severe for photochemical simulations and makes SF-TDDFT challenging to apply in a general way for NMD. In our experience, when starting from a triplet reference configuration, states that manifest as singlets upon vertical excitation $\left(\left\langle\hat{S}^{2}\right\rangle \approx 0\right)$ quickly become highly mixed as the molecule moves away from the Frank-Condon region (typically $\left\langle\hat{S}^{2}\right\rangle \approx 1$, in atomic units). As a result, it becomes difficult to distinguish singlets from triplets, and to proceed without human intervention requires some kind of a state-tracking procedure. ${ }^{12,38,39}$ Although some NMD simulations using SF-TDDFT have been reported using this approach, ${ }^{21,39-46}$ a more reliable remedy is to remove the spin contamination directly. This can be done either exactly, by construction, ${ }^{9-12}$ or else approximately, ${ }^{47-51}$ but these procedures are not yet in widespread use.

Fortunately, photoisomerization of the PSB3 molecule that is considered herein involves only the two lowest singlet states. ${ }^{22}$ Instead of using a fully spin-adapted version of SF-TDDFT, ${ }^{12}$ for which the analytic gradient is not yet available, we instead aim to obtain only approximately spin-pure $S_{0}$ and $S_{1}$ states. To achieve this, we simply enforce that the two open-shell Slater determinants in Fig. 2(b) must form a singlet configuration state function when solving the SF-TDDFT eigenvalue equations, meaning that their coefficients must be equal in magnitude and opposite in sign. For PSB3, the $\mathrm{S}_{0}$ and $\mathrm{S}_{1}$ states that we obtain in this way typically have $\left\langle\hat{S}^{2}\right\rangle<0.5$ and are thus easy to distinguish from triplet states. As discussed below, we do observe some exceptions, in which $\mathrm{S}_{1}$ is significantly spin contaminated (with $\left\langle\hat{S}^{2}\right\rangle \approx 1$ ); this occurs in cases where one of the single bonds in PSB3 is significantly twisted. However, the manifold of excited states is sparse enough that we are nevertheless able to perform consistent NMD simulations via trajectory surface-hopping, without resort to state-tracking algorithms.

\section{COMPUTATIONAL DETAILS}

NMD simulations for trans-PSB3 were performed using the augmented fewest-switches surface hopping (aFSSH) algorithm, ${ }^{52-54}$ a modification of the original fewest-switches algorithm ${ }^{55}$ to account for decoherence effects. ${ }^{54}$ Separate simulations were performed using LR-TDDFT (both with and without the TDA) and also SF-TDDFT. For the LR-TDDFT simulations we used the $\omega$ B97X functional, ${ }^{56}$ and for SF-TDDFT we used BH\&HLYP. ${ }^{57,58}$ (Early benchmarks of SF-TDDFT demonstrated that a functional with $50 \%$ exact exchange works well, ${ }^{7}$ a fact that was later justified theoretically, ${ }^{59}$ and BH\&HLYP has become the de facto standard for use with SF-TDDFT. ${ }^{21,39-45,47-50,60}$ ) The $6-31 \mathrm{G}^{*}$ basis set was used for all aFSSH simulations. All calculations were performed using a locally-modified copy of Q-Chem, 
v. $4 .{ }^{61}$

A set of 200 aFSSH trajectories were computed at each level of theory. The initial nuclear configurations and momenta were sampled according to the Wigner distribution for the quantum harmonic oscillator, with harmonic frequencies and normal modes obtained from ground-state MP2 calculations. At $t=0$ in the simulations, the $\mathrm{S}_{1}$ state is populated at the ground-state geometry and each trajectory was then propagated in time for 9,000 a.u. $(\approx 218 \mathrm{fs})$, using a time step of 20 a.u. $(\approx 0.484 \mathrm{fs})$. These trajectories are intended to model a real photoexcitation of PSB3. For reasons described below, we also propagated a second batch of 200 trajectories (at each of three levels of theory) in which the central $\mathrm{C}_{2}=\mathrm{C}_{3}$ bond is twisted by $25^{\circ}$ at $t=0$. These "activated" trajectories were propagated in the same way and analyzed separately.

Nonadiabatic coupling vectors are required for the aFSSH simulations and are available in analytic form in Q-Chem, ${ }^{6,29}$ for both LR-TDDFT and SF-TDDFT. Whereas calculation of the derivative couplings is sometimes (incorrectly) perceived as being significantly more expensive than analytic gradients, in reality the calculation of $\mathbf{h}_{I J}$ for TDDFT adds only about $10 \%$ overhead on top of the cost of computing the gradients $\hat{\nabla}_{\mathbf{R}} E_{I}(\mathbf{R})$ and $\hat{\nabla}_{\mathbf{R}} E_{J}(\mathbf{R}){ }^{6,20}$

\section{RESULTS AND DISCUSSION}

\section{A. Potential energy surfaces along the reaction pathways}

Gozem et al. ${ }^{25}$ have reported photoisomerization reaction pathways for PSB3 at the CASSCF and CASPT2 levels of theory. These pathways connect the cis and trans isomers of PSB3 with a CX seam between the $\mathrm{S}_{0}$ and $\mathrm{S}_{1}$ states. The most important geometric parameters that characterize these pathways are the bond-length alternation (BLA) coordinate, the $\mathrm{C}_{1}-\mathrm{C}_{2}$ single-bond torsion, and the $\mathrm{C}_{2}=\mathrm{C}_{3}$ double-bond torsion. [See Fig. 1(b) for the PSB3 structure.] The reaction pathway computed at the CASSCF level shows a decrease in the BLA as the molecule moves away from Frank-Condon region on the $\mathrm{S}_{1}$ potential surface. Following that, the central double bond $\left(\mathrm{C}_{2}=\mathrm{C}_{3}\right)$ starts to twist and the BLA coordinate increases again until the $\mathrm{CX}$ region is reached, at which point a nonadiabatic transition returns the system to the ground state. For the CASPT2 reaction path, however, the same BLA change in the Frank-Condon region leads to a shallow local minimum (called "MIN trans" in what follows), which corresponds to a structure with a rotated $\mathrm{C}_{1}-\mathrm{C}_{2}$ single bond. ${ }^{25}$ According to the CASPT2 calculations, the $\mathrm{C}_{2}=\mathrm{C}_{3}$ torsion is activated by overcoming a small barrier.

We performed single-point potential energy scans along the same CASSCF and CASPT2 reaction pathways that were reported in Ref. 25, using SF-TDDFT and
LR-TDDFT. The results are compared with the energies calculated from multireference configuration interaction singles and doubles (MRCISD) with the Davidson correction (MRCISD+Q), taken from Ref. 25. The $S_{0}$ and $\mathrm{S}_{1}$ potential surfaces along the CASSCF and CASPT2 pathways are depicted in Fig. 3.

For both pathways, energy profiles computed using SF-TDDFT with the BH\&HLYP functional (called "SFBH\&HLYP" hereafter) agree well with the MRCISD $+\mathrm{Q}$ results, except that SF-BH\&HLYP predicts a larger energy gap in the CX regions, due to the fact that the intersection seams obtained by these two methods are located in somewhat different regions of coordinate space. This is not surprising as MRCISD+Q fails to give the correct topology of the CX, because the Davidson correction $(+Q)$ modifies the energy but not the wave function. ${ }^{25}$

In the present study, the $\omega$ B97X functional is used for the LR-TDDFT calculations and we abbreviate this as LR- $\omega$ B97X. Previously, Casida and co-workers compared the performance of different density functionals for the out-of-plane relaxations of the retinal protonated Schiff base models PSB4 and PSB5. ${ }^{62}$ Taking the CASPT2 results as the reference, they found that a particular longrange corrected (LRC) functional, LRC- $\mu \mathrm{BLYP},{ }^{63,64}$ outperformed other functionals including $\omega \mathrm{B} 97 \mathrm{X}$. For the PSB3 reaction pathways considered here, however, we find that the performance of $\omega \mathrm{B} 97 \mathrm{X}$ and LRC- $\mu \mathrm{BLYP}$ is almost identical. This lends some credence to the idea that the LR-TDDFT results reported here are unlikely to change qualitatively if other functionals commonly used for LR-TDDFT are substituted in place of $\omega$ B97X.

As shown in Fig. 3, LR-TDDFT energetics agree well with the MRCISD $+\mathrm{Q}$ results within the $\mathrm{CX}$ region but large differences are found close to the Franck-Condon regions, especially when the TDA is invoked. For the CASSCF pathway, LR- $\omega$ B97X predicts a local minimum close to the Franck-Condon point that does not exist in either the SF-BH\&HLYP or the MRCISD+Q calculations. In view of the trajectory simulations reported below, we believe that this local minimum connects directly to the $\mathrm{C}_{1}-\mathrm{C}_{2}$ single bond torsion reaction pathway. Energy profiles in Fig. 3(b) show that the energy of the $\mathrm{MIN}_{\text {trans }}$ configuration is lower at the LR- $\omega$ B97X level of theory as compared to other methods examined here, meaning that LR- $\omega \mathrm{B} 97 \mathrm{X}$ trajectories must overcome a larger barrier to activate the $\mathrm{C}_{2}=\mathrm{C}_{3}$ double bond torsion reaction.

\section{B. Stationary points, vertical excitation energies, and topography of conical intersections}

Whereas the calculations discussed above were performed at CASSCF and CASPT2 geometries, we have also optimized the geometries of the local minima on $\mathrm{S}_{0}$ and $\mathrm{S}_{1}$, and the $\mathrm{S}_{1} / \mathrm{S}_{0}$ minimum-energy crossing points (MECPs), using SF-TDDFT, LR-TDDFT, and LR-TDDFT/TDA; results are depicted in Fig. 4. For the 
(a) CASSCF pathway

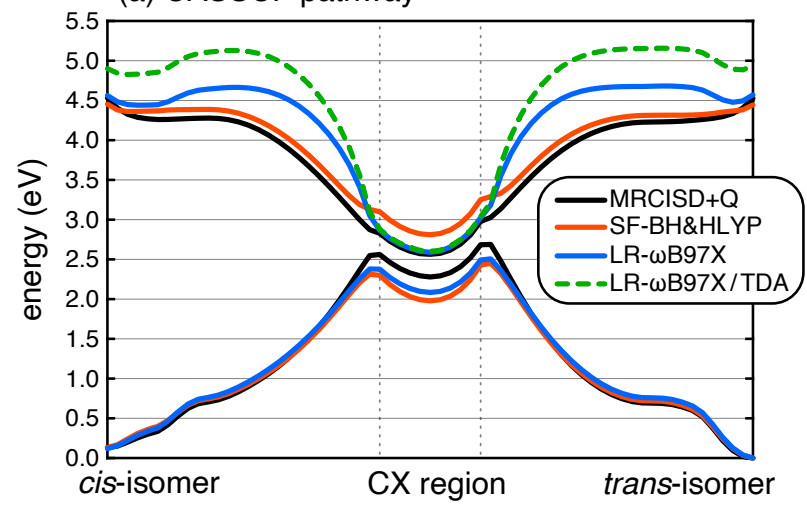

(b) CASPT2 pathway

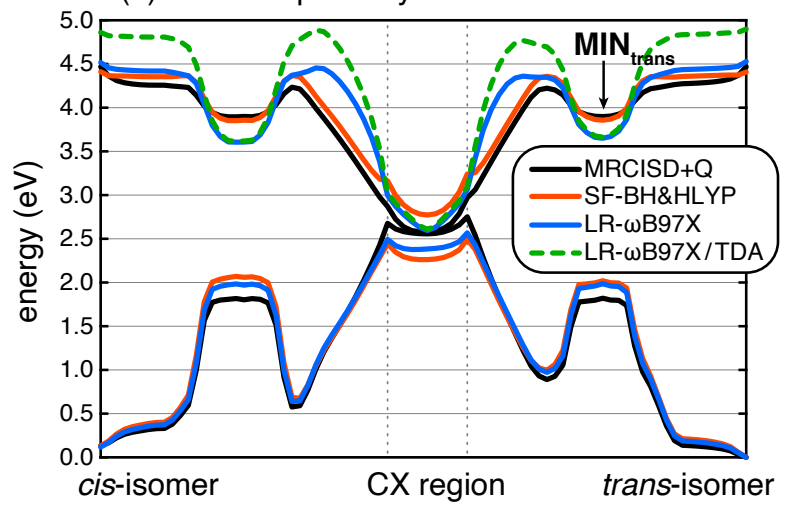

FIG. 3: Potential energy scans along the (a) CASSCF or (b) CASPT2 photoisomerization pathway PSB3. Both the pathway geometries and the MRCISD+Q energies are from Ref. 25. All calculations employ the 6-31G* basis set.

local minima these three methods predict almost identical geometries. Automated optimization of MECPs fails at the LR- $\omega$ B97X level, due to triplet instabilities, there is no such difficulty when the TDA is applied. (Other results suggest that triplet instabilities are commonplace in bond-breaking regions of the potential energy surface, making the TDA "a practical necessity" for photochemical simulations. ${ }^{65}$ ) MECP geometries optimized at the LR- $\omega$ B97X/TDA and SF-BH\&HLYP levels agree with each other very well, and also agree qualitatively with MS-CASPT2 results. ${ }^{66}$

Table I summarizes the relative energies of the various critical points shown in Fig. 4. Overall, the energies computed at the three DFT levels agree qualitatively with each other and with multistate (MS-)CASPT2 results. The DFT methods overestimate the vertical excitation energy as compared to MS-CASPT2, especially in the case of LR- $\omega$ B97X/TDA. Among the three MECPs considered here, all methods predict that the one with a twisted $\mathrm{C}_{2}=\mathrm{C}_{3}$ double bond is lowest in energy; this is the structure labeled $\operatorname{MECP}\left(\mathrm{C}_{2} \mathrm{C}_{3}\right)$ in Fig. 4 and Table I. The SF-BH\&HLYP method places the three MECP a bit higher in energy as compared to LR- $\omega$ B97X/TDA. However, if one considers the energetics relative to the $\mathrm{S}_{1}$ state at Franck-Condon region, the SF-BH\&HLYP results are more consistent with the MS-CASPT2 results.

We also examined the topography around the CXs, which is believed to have correlations with the efficiency of nonadiabatic transitions. Here, we only consider $\operatorname{MECP}\left(\mathrm{C}_{2} \mathrm{C}_{3}\right)$ because most of the nonadiabatic $\mathrm{S}_{1} \rightarrow \mathrm{S}_{0}$ transitions in NMD simulations discussed below occur near this particular CX. Yarkony ${ }^{67}$ has introduced several parameters that characterize the topography of a CX. Denoting the lengths of the branching-plane vectors introduced in Section II A as $g=\|\mathbf{g}\|$ and $h=\|\mathbf{h}\|$, these topographical parameters include

$$
d_{g h}=\sqrt{g^{2}+h^{2}}
$$
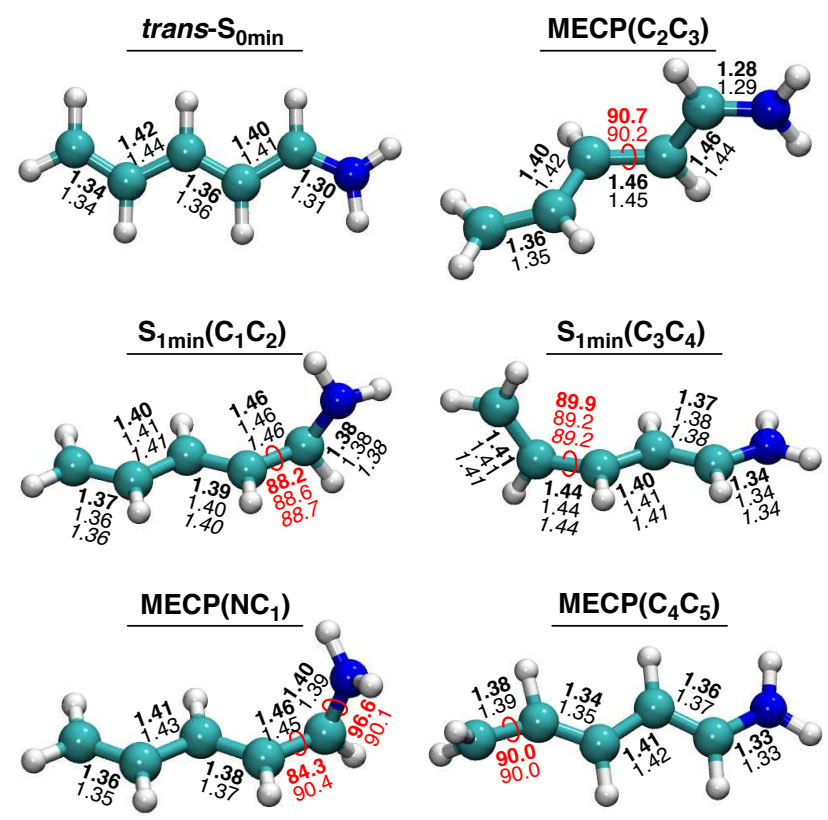

FIG. 4: Critical-point structures of PSB3 including $\mathrm{S}_{0}$ and $\mathrm{S}_{1}$ minima and the $\mathrm{S}_{1} / \mathrm{S}_{0} \mathrm{MECP}$, optimized using SFBH\&HLYP (geometric parameters in bold), LR- $\omega$ B97X/TDA (parameters in plain font), and LR- $\omega$ B97X (parameters in italics). The bond lengths (in Ångstroms) are labeled in black and the twist angles (in degrees) are in red. MECP geometries were not optimized using full LR-TDDFT but rather only with the TDA, due to triplet instability problems.

and

$$
\Delta_{g h}=\frac{g^{2}-h^{2}}{d_{g h}^{2}}
$$

which describe the sharpness and asymmetry of the CX. Defining a "seam coordinate"

$$
\mathbf{s}_{I J}=\frac{1}{2} \hat{\nabla}_{\mathbf{R}}\left[E_{I}(\mathbf{R})+E_{J}(\mathbf{R})\right]
$$


TABLE I: Relative energies (in eV) at stationary points of PSB3. ${ }^{a}$

\begin{tabular}{|c|c|c|c|c|c|c|c|c|}
\hline \multirow{2}{*}{ Structures } & \multicolumn{2}{|c|}{ SF-BH\&HLYP ${ }^{b}$} & \multicolumn{2}{|c|}{ LR- $\omega$ B97X } & \multicolumn{2}{|c|}{$\overline{\text { LR- } \omega \text { B97X/TDA }}$} & \multicolumn{2}{|c|}{$\mathrm{CASPT}^{c}$} \\
\hline & $\mathrm{S}_{0}$ & $\mathrm{~S}_{1}$ & $\mathrm{~S}_{0}$ & $\mathrm{~S}_{1}$ & $\mathrm{~S}_{0}$ & $\mathrm{~S}_{1}$ & $\mathrm{~S}_{0}$ & $\mathrm{~S}_{1}$ \\
\hline$\overline{\operatorname{trans}}-\mathrm{S}_{0 \min }$ & $0.00(0.04)$ & $4.48(0.17)$ & 0.00 & 4.59 & 0.00 & 4.95 & 0.00 & 4.26 \\
\hline $\operatorname{MECP}\left(\mathrm{C}_{2} \mathrm{C}_{3}\right)$ & $3.07(0.03)$ & $3.07(0.02)$ & & & 2.61 & 2.61 & & 2.61 \\
\hline $\operatorname{MECP}\left(\mathrm{NC}_{1}\right)$ & $4.29(0.02)$ & $4.29(0.08)$ & & & 4.09 & 4.10 & & 3.81 \\
\hline $\operatorname{MECP}\left(\mathrm{C}_{4} \mathrm{C}_{5}\right)$ & $3.31(0.05)$ & $3.31(0.05)$ & & & 3.22 & 3.22 & & 3.59 \\
\hline $\mathrm{S}_{1 \mathrm{~min}}\left(\mathrm{C}_{1} \mathrm{C}_{2}\right)$ & $1.85(0.06)$ & $3.86(0.02)$ & 1.78 & 3.63 & 1.77 & 3.64 & & \\
\hline $\mathrm{S}_{1 \min }\left(\mathrm{C}_{3} \mathrm{C}_{4}\right)$ & $1.00(0.01)$ & $3.82(1.01)$ & 0.92 & 4.10 & 0.92 & 4.11 & & 3.69 \\
\hline
\end{tabular}

${ }^{a}$ The $6-31 \mathrm{G}^{*}$ basis set is used for all calculations.

${ }^{b}$ The value of $\left\langle\hat{S}^{2}\right\rangle$ (in atomic units) is given in parenthesis.

${ }^{c}$ SA3-MS-CASPT2 $(6,6) / 6-31 G^{*}$ results from Ref. 66.

TABLE II: Parameters characterizing the topography at the $\operatorname{MECP}\left(\mathrm{C}_{2} \mathrm{C}_{3}\right)$ structure of PSB3, in atomic units.

\begin{tabular}{|c|c|c|c|c|}
\hline \multirow{3}{*}{ Parameter } & \multicolumn{3}{|c|}{ TDDFT $^{a}$} & \multirow{3}{*}{$\mathrm{CASSCF}^{b}$} \\
\hline & \multirow{2}{*}{$\begin{array}{c}\text { SF- } \\
\text { BH\&HLYP }\end{array}$} & \multicolumn{2}{|c|}{ LR- $\omega$ B97X } & \\
\hline & & full $^{c}$ & TDA & \\
\hline$\overline{d_{g h}}$ & 0.10 & $\overline{0.49}$ & 0.36 & 0.13 \\
\hline$\Delta_{g h}$ & 0.15 & 1.00 & 1.00 & 0.29 \\
\hline$s_{x}$ & -0.05 & 0.13 & 0.11 & -0.02 \\
\hline$s_{y}$ & -0.13 & -0.15 & 0.16 & 0.12 \\
\hline$s_{x} / g$ & -0.67 & 0.26 & 0.32 & -0.16 \\
\hline$s_{y} / h$ & -2.08 & -7.67 & $>1000$ & 1.63 \\
\hline
\end{tabular}

${ }^{a} 6-31 \mathrm{G}^{*}$ basis set

${ }^{b} \mathrm{SA} 3-\mathrm{CASSCF}(6,6) / 6-31 \mathrm{G}$ results from Ref. 66.

${ }^{c}$ Using the geometry from Fig. $5 \mathrm{~b}$.

one may then define dimensionless tilt parameters

$$
\begin{aligned}
& s_{x}=\left(\mathbf{s}_{I J} \cdot \mathbf{g}_{I J}\right) / g \\
& s_{y}=\left(\mathbf{s}_{I J} \cdot \mathbf{h}_{I J}\right) / h .
\end{aligned}
$$

Larger values of $s_{x}$ or $s_{y}$ indicating sloped rather than peaked character along the direction of corresponding branching-plane vector $\left(\mathbf{g}_{I J}\right.$ or $\mathbf{h}_{I J}$, respectively). If $s_{x}=s_{y}=\Delta_{g h}=0$, the CX is vertical and cylindrically symmetric. ${ }^{67}$ Topographical parameters for the $\operatorname{MECP}\left(\mathrm{C}_{2} \mathrm{C}_{3}\right)$ point of $\mathrm{C}_{5} \mathrm{H}_{6} \mathrm{NH}_{2}^{+}$are listed in Table II.

The two LR-TDDFT methods predict much larger values of $d_{g h}$ as compared to either SF-BH\&HLYP or CASSCF, indicating a much more sharply peaked topography near the CX. Meanwhile, $\Delta_{g h} \approx 1$ for the LRTDDFT methods, indicating that the CX is strongly asymmetric. Along with the very large values of $s_{y} / h$, this indicates significant slope along the $\mathbf{h}$ direction, which is a direct consequence of nearly vanishing coupling between $S_{0}$ and $S_{1}$ in LR-TDDFT. The same conclusion can be reached by examining the $\mathbf{g}$ and $\mathbf{h}$ vectors, which are plotted for $\operatorname{MECP}\left(\mathrm{C}_{2} \mathrm{C}_{3}\right)$ in Fig. 5. To have a point of comparison at the LR- $\omega$ B97X level, where the appearance of triplet instabilities precludes a full optimization of the MECP, we started in that case from the $\operatorname{MECP}\left(\mathrm{C}_{2} \mathrm{C}_{3}\right)$ structure located using LR- $\omega \mathrm{B} 97 \mathrm{X} / \mathrm{TDA}$ and minimized along the energy gap until an instabil- (a) SF-BHHLYP
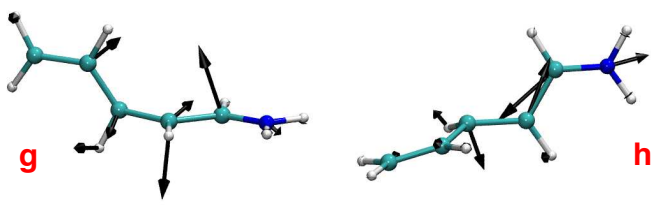

(b) LR- $\omega$ B97X
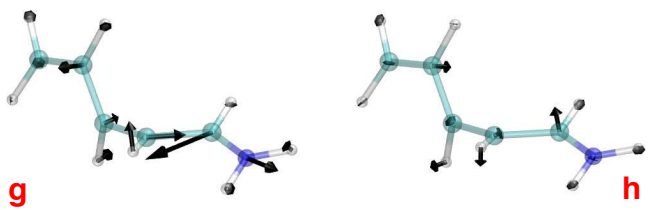

(c) LR- $\omega B$ B7X/TDA
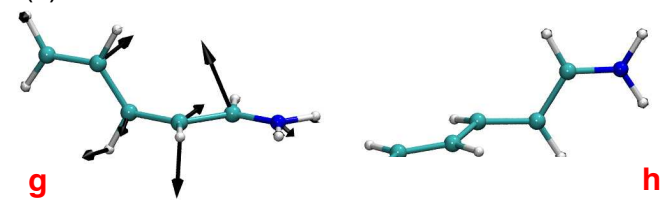

FIG. 5: Gradient difference vectors (g, on the left) and nonadiabatic coupling vectors (h, on the right) at the $\operatorname{MECP}\left(\mathrm{C}_{2} \mathrm{C}_{3}\right)$ structure of PSB3, computed using (a) SFBH\&HLYP, (b) LR- $\omega$ B97X, and (c) LR- $\omega$ B97X/TDA. Difficulties with triplet instabilities preclude full optimization of the MECP at the LR- $\omega$ B97X level, but the structure in (b) has an $\mathrm{S}_{1} / \mathrm{S}_{0}$ gap of only $0.0072 \mathrm{eV}$ and is likely close to the true MECP. For readability, the $\mathbf{g}$ vectors in (b) and (c) have been scaled by 0.1 and 0.2 , respectively, as the LR-TDDFT g-vectors are significantly larger than that obtained at the SF-BH\&HLYP level.

ity appeared. The resulting structure has an energy gap $<0.01 \mathrm{eV}$ and is likely very close to a MECP structure.

The $\mathbf{g}$ vectors computed using LR-TDDFT are more than ten times greater in length than those obtained with SF-BH\&HLYP, indicating significantly larger slope along the potential surface in the $\mathbf{g}$ direction when using LRTDDFT. Small magnitude of the $\mathbf{h}$ vectors, especially when the TDA is applied, is a sign of nearly zero interaction between the reference state and the excited state. In contrast, SF-BH\&HLYP predicts topography around 
(a) photoexcitation initial conditions

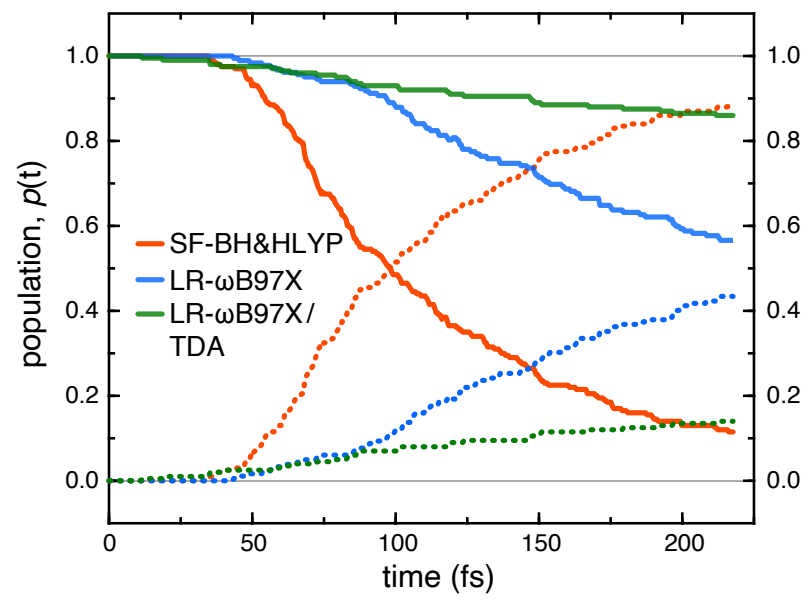

(b) activated initial conditions

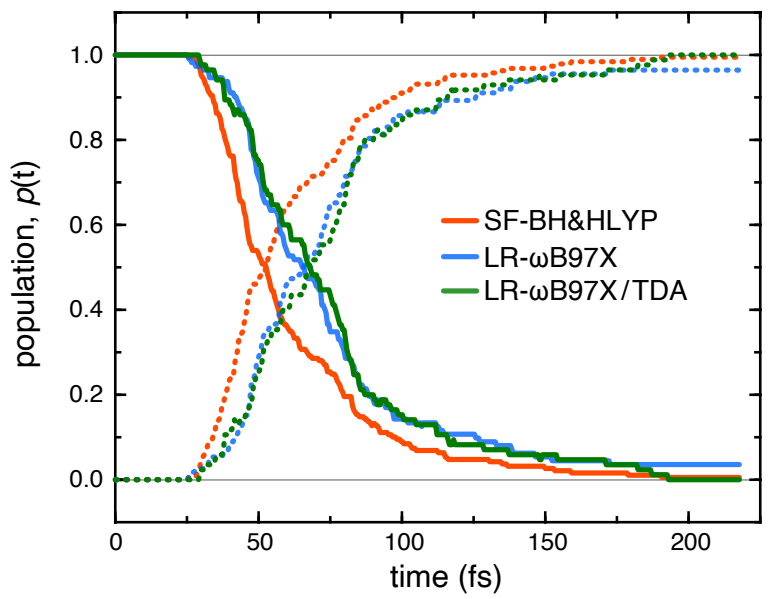

FIG. 6: Populations of the $\mathrm{S}_{0}$ state (dotted curves) and the $\mathrm{S}_{1}$ state (solid curves) for the photoisomerization dynamics of PSB3, averaged over aFSSH trajectories from two different sets of initial conditions. (a) Initial conditions simulating $\mathrm{S}_{0} \rightarrow \mathrm{S}_{1}$ photoexcitation, with internal coordinates and velocities selected from a ground-state Wigner distribution and placed on the $\mathrm{S}_{1}$ surface at $t=0$. Populations $p(t)$ are averages over 200 trajectories at each level of theory. (b) "Activated" trajectories, in which the $\mathrm{C}_{2}=\mathrm{C}_{3}$ bond was rotated by $25^{\circ}$ for each of the initial structures in (a), and furthermore any trajectories that did not proceed through $\mathrm{MECP}\left(\mathrm{C}_{2} \mathrm{C}_{3}\right)$ were removed.

$\operatorname{MECP}\left(\mathrm{C}_{2} \mathrm{C}_{3}\right)$ that is similar to CASSCF, as can be seen from the parameters listed in Table II.

\section{Trajectory surface hopping simulations}

\section{Photoexcitation initial conditions}

We next discuss results of the aFSSH simulations of PSB3 starting from photoexcitation initial conditions, in which internal coordinates and velocities are sampled from a ground-state Wigner distribution and initiated on the $\mathrm{S}_{1}$ state at $t=0$. Figure $6(\mathrm{a})$ shows the timedependent populations of the $\mathrm{S}_{0}$ and $\mathrm{S}_{1}$ states averaged over 200 trajectories at each of the three levels of TDDFT that were introduced above. The $\mathrm{S}_{1}$ population exhibits a delayed exponential decay, meaning that the system remains in the $\mathrm{S}_{1}$ state for a certain amount of time before the population begins to decrease exponentially. The lifetime of the $\mathrm{S}_{1}$ state can be obtained by fitting the population $p(t)$ using the function

$$
p(t)= \begin{cases}1, & t<\tau_{\mathrm{d}} \\ e^{-\left(t-\tau_{\mathrm{d}}\right) / \tau_{\mathrm{e}},} & t \geq \tau_{\mathrm{d}}\end{cases}
$$

where $\tau_{\mathrm{d}}$ represents the initial delay time for the system remaining in the $\mathrm{S}_{1}$ state and $\tau_{\mathrm{e}}$ is the time constant for the exponential decay. The overall lifetime is $\tau_{\mathrm{d}}+\tau_{\mathrm{e}}$, which is technically the time required for the $\mathrm{S}_{1}$ population to drop to $1 / e$ of its initial value $p(0)=1$. We fit the data in Fig. 6(a) to this functional form, and the time constants thus obtained (for each level of theory) are listed in Table III. Uncertainties listed in the table were obtained using the bootstrap method. ${ }^{68}$
The two LR-TDDFT methods predict much longer lifetimes for the $\mathrm{S}_{1}$ state as compared to SF-BH\&HLYP and CASSCF. In the latter two methods, most of the trajectories are trapped at the local minimum $\mathrm{S}_{1 \text { min }}\left(\mathrm{C}_{1} \mathrm{C}_{2}\right)$, where the molecules have to overcome a large barrier before the conical seam at $\operatorname{MECP}\left(\mathrm{C}_{2} \mathrm{C}_{3}\right)$ can be reached; see Fig. 3(b). That LR- $\omega$ B97X/TDA predicts a longer lifetime for the $\mathrm{S}_{1}$ state as compared to LR- $\omega \mathrm{B} 97 \mathrm{X}$ is also consistent with the larger barrier predicted by the former as compared to the latter.

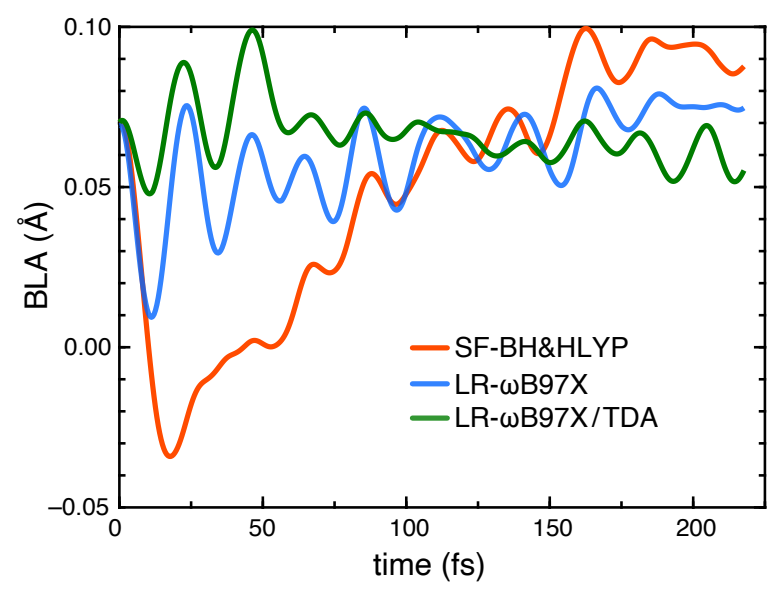

FIG. 7: Bond length alternation (BLA) coordinate as a function of time for PSB3 photoisomerization dynamics, averaged over 200 trajectories (starting from photoexcitation initial conditions) at each of three levels of theory. These coordinate dynamics correspond to the population dynamics shown in Fig. 6(a). 
TABLE III: Time constants $\tau_{\mathrm{d}}$ and $\tau_{\mathrm{e}}$ and total lifetime of the $\mathrm{S}_{1}$ state of PSB3 (all in fs), obtained by fitting the population data in Fig. 6 using Eq. (20).

\begin{tabular}{|c|c|c|c|c|c|c|}
\hline \multirow[t]{2}{*}{$\overline{\text { Method }}$} & \multicolumn{3}{|c|}{ 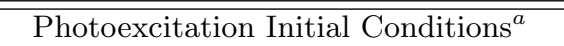 } & \multicolumn{3}{|c|}{ 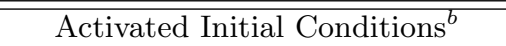 } \\
\hline & $\tau_{\mathrm{d}}$ & $\overline{\tau_{\mathrm{e}}}$ & lifetime & $\tau_{\mathrm{d}}$ & $\tau_{\mathrm{e}}$ & lifetime \\
\hline SF-BH\&HLYP & $35.1 \pm 1.4$ & $89.6 \pm 6.3$ & $124.8 \pm 6.1$ & $26.2 \pm 1.4$ & $34.4 \pm 2.7$ & $60.6 \pm 2.4$ \\
\hline LR- $\omega \mathrm{B} 97 \mathrm{X}$ & $44.1 \pm 2.4$ & $320.6 \pm 42.4$ & $364.8 \pm 42.2$ & $26.0 \pm 1.5$ & $48.5 \pm 3.4$ & $74.5 \pm 3.5$ \\
\hline LR $-\omega \mathrm{B} 97 \mathrm{X} / \mathrm{TDA}$ & $15.8 \pm 7.5$ & $1264 \pm 269$ & $1280 \pm 269$ & $29.4 \pm 1.0$ & $45.3 \pm 3.9$ & $74.7 \pm 3.9$ \\
\hline $\mathrm{CASPT}^{c}$ & $\approx 50$ & & $\approx 150$ & & & \\
\hline
\end{tabular}

${ }^{a}$ Population data plotted in Fig. 6(a).

${ }^{b}$ Population data plotted in Fig. 6(b).

${ }^{c}$ SA3-MS-CASPT2 $(6,6) / 6-31 \mathrm{G}$ results from Ref. 66.

(a) $\mathrm{Y}\left(\mathrm{C}_{1} \mathrm{C}_{2} \mathrm{C}_{3} \mathrm{C}_{4}\right)$
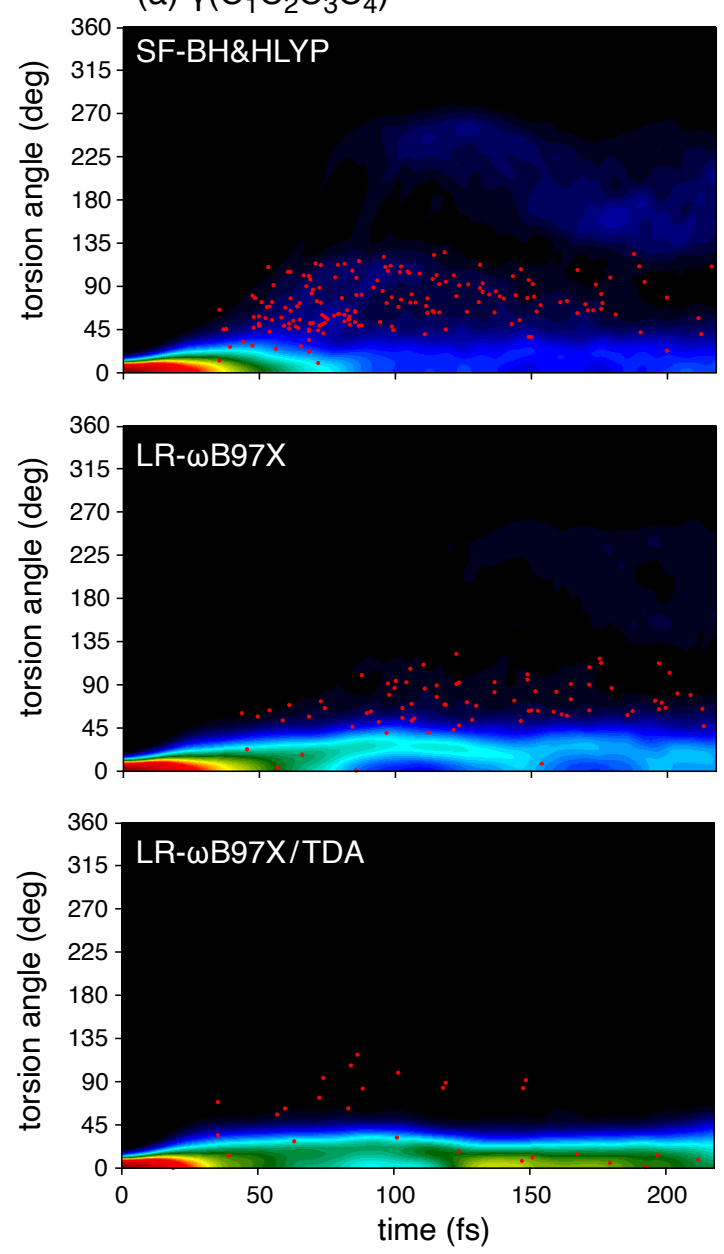

(b) $\mathrm{Y}\left(\mathrm{NC}_{1} \mathrm{C}_{2} \mathrm{C}_{3}\right)$
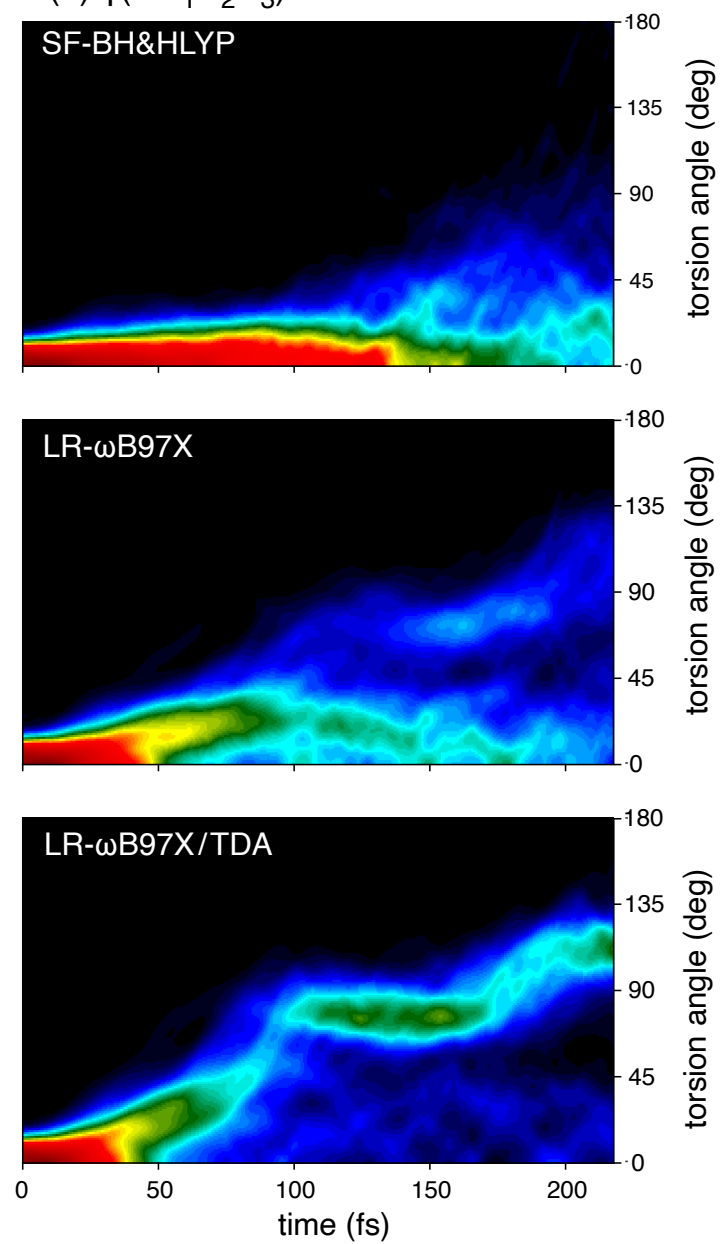

FIG. 8: Distributions of the torsion angles (a) $\gamma\left(\mathrm{C}_{1} \mathrm{C}_{2} \mathrm{C}_{3} \mathrm{C}_{4}\right)$ and (b) $\gamma\left(\mathrm{NC}_{1} \mathrm{C}_{2} \mathrm{C}_{3}\right)$ for aFSSH simulations of PSB3, each at three different levels of theory. These trajectories correspond to "photoexcitation" initial conditions in which coordinates and velocities sampled from ground-state harmonic oscillators are propagated on the $\mathrm{S}_{1}$ surface starting from $t=0$. [The corresponding population dynamics are plotted in Fig. 6(a).] The probability density ranges from 0 to 1 as the color varies from black to red. Red dots in (a) indicate hopping events.

In Figure 7, we plot the averaged BLA coordinate values during the simulations. For LR- $\omega$ B97X, either with and without the TDA, the BLA does not decrease to an extent such that the reaction coordinate (corresponding to $\mathrm{C}_{2}=\mathrm{C}_{3}$ torsion) can be activated, therefore only a few of the trajectories manage to return to the $\mathrm{S}_{0}$ state via nonadiabatic transitions at $\operatorname{MECP}\left(\mathrm{C}_{2} \mathrm{C}_{3}\right)$. This is more clear in Fig. 8(a), where the distribution of the torsion angle around $\mathrm{C}_{2}=\mathrm{C}_{3}$ is plotted for each of the three DFT methods. In the case of SF-BH\&HLYP, about $90 \%$ of the trajectories returned to the $\mathrm{S}_{0}$ state on the timescale of the simulation, with $56 \%$ of them forming the cis pho- 
(a) $\mathrm{Y}\left(\mathrm{C}_{1} \mathrm{C}_{2} \mathrm{C}_{3} \mathrm{C}_{4}\right)$
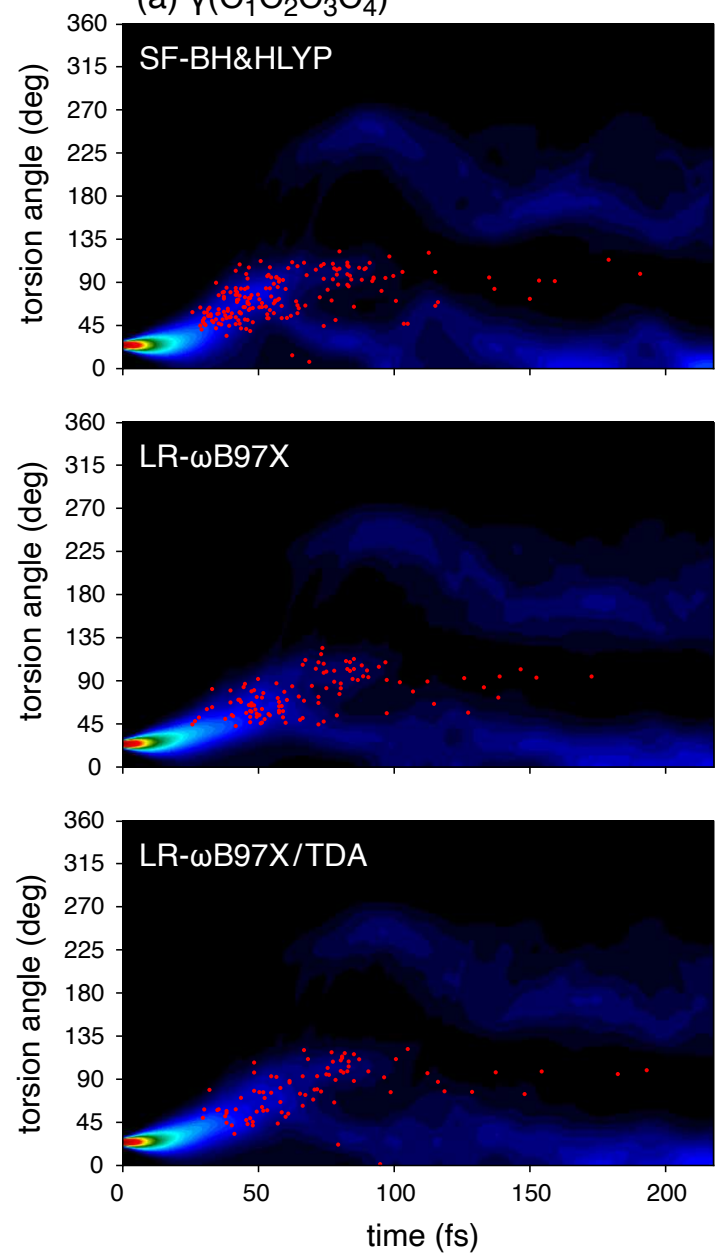

(b) $\mathrm{Y}\left(\mathrm{NC}_{1} \mathrm{C}_{2} \mathrm{C}_{3}\right)$
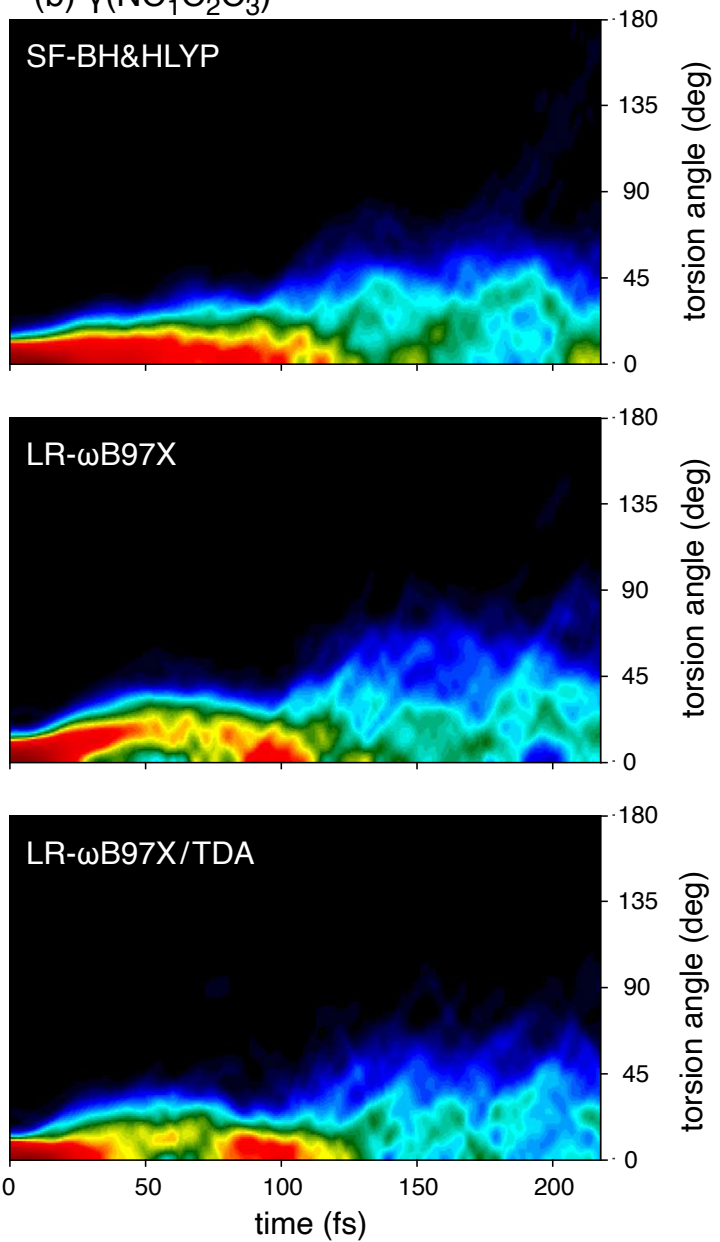

FIG. 9: Distributions of the torsion angles (a) $\gamma\left(\mathrm{C}_{1} \mathrm{C}_{2} \mathrm{C}_{3} \mathrm{C}_{4}\right)$ and (b) $\gamma\left(\mathrm{NC}_{1} \mathrm{C}_{2} \mathrm{C}_{3}\right)$ for aFSSH simulations of PSB3, each at three different levels of theory, starting from "activated" initial conditions in which $\gamma\left(\mathrm{C}_{1} \mathrm{C}_{2} \mathrm{C}_{3} \mathrm{C}_{4}\right)$ is twisted by $25^{\circ}$ at $t=0$, and retaining only those trajectories that pass through $\operatorname{MECP}\left(\mathrm{C}_{2} \mathrm{C}_{3}\right)$. The corresponding population dynamics are plotted in Fig. 6(b). The probability density ranges from 0 to 1 as the color varies from black to red. Red dots in (a) indicate hopping events.

toproduct. For LR- $\omega$ B97X, however, only $40 \%$ of the trajectories underwent $\mathrm{S}_{1} \rightarrow \mathrm{S}_{0}$ deactivation within the 218 fs timescale that we simulate, and amongst those that do return to the ground state, $43 \%$ relaxed to the cis isomer. Even fewer transitions were observed at the LR- $\omega$ B97X/TDA level, which amounts to $14 \%$ of the total number of trajectories, and $25 \%$ of the nonadiabatic transitions lead to the cis isomer. If we further plot the distribution of the torsion angle for the $\mathrm{C}_{1}-\mathrm{C}_{2}$ single bond [Fig. 8(b)], we find that most of the trajectories computed by the two LR-TDDFT methods follow the reaction pathways of $\mathrm{C}_{1}-\mathrm{C}_{2}$ twisting, especially at the LR$\omega \mathrm{B} 97 \mathrm{X} / \mathrm{TDA}$ level. This corresponds either to trapping at $\mathrm{S}_{1 \text { min }}\left(\mathrm{C}_{1} \mathrm{C}_{2}\right)$ or hopping at $\operatorname{MECP}\left(\mathrm{NC}_{1}\right)$. The latter explains the observation of fewer cis photoproducts in the LR-TDDFT trajectories as well as the smaller delay time $\tau_{\mathrm{d}}$, as compared to SF-BH\&HLYP simulations.

\section{Activated initial conditions}

The main goal of this work is to study the effect of incorrect CX topography on NMD simulations within the framework of TDDFT, and for side-by-side comparison of LR- and SF-TDDFT we would ideally like to have a set of trajectories from either method that undergo the same reaction pathway, namely, nonadiabatic transition at $\operatorname{MECP}\left(\mathrm{C}_{2} \mathrm{C}_{3}\right)$. In order to achieve this, we have performed an entirely new set of aFSSH simulations starting from "activated" initial conditions. By this we mean that the same set of starting coordinates and velocities was used as in the simulations described above (where they were sampled from ground-state harmonic oscillators), but in the present case the $\mathrm{C}_{2}=\mathrm{C}_{3}$ bond was twisted by an additional $25^{\circ}$ in each individual starting structure. The can be seen in the plots of the time-dependent torsion angle $\gamma\left(\mathrm{C}_{1} \mathrm{C}_{2} \mathrm{C}_{3} \mathrm{C}_{4}\right)$, which are 
presented in Fig. 9(a), where it can be seen that the distributions at $t=0$ are peaked narrowly around $\gamma=25^{\circ}$, unlike the corresponding "unactivated" distributions in Fig. 8(a), for which $\gamma \approx 0$ at $t=0$.

Even with this activation step, we find that quite a few of the LR-TDDFT trajectories fail to proceed through $\operatorname{MECP}\left(\mathrm{C}_{2} \mathrm{C}_{3}\right)$ on the timescale that we simulate. Many of them become trapped at $\mathrm{S}_{1 \mathrm{~min}}\left(\mathrm{C}_{1} \mathrm{C}_{2}\right)$, and a few others access the ground state via either $\operatorname{MECP}\left(\mathrm{NC}_{1}\right)$ or $\operatorname{MECP}\left(\mathrm{C}_{4} \mathrm{C}_{5}\right)$. In order to have a side-by-side comparison of similar dynamical pathways at different levels of theory, we discarded all but the trajectories that return to the ground state via $\operatorname{MECP}\left(\mathrm{C}_{2} \mathrm{C}_{3}\right)$. This corresponds to 189 trajectories retained for the SF-BH\&HLYP simulation, 112 retained for LR- $\omega$ B97X, and 85 for LR$\omega \mathrm{B} 97 \mathrm{X} / \mathrm{TDA}$. All of the analysis below corresponds to these subsets of the 200 trajectories. Population decays from $S_{1}$ are plotted in Fig. 6(b) for this subset of trajectories, and then $p(t)$ was fit to Eq. (20). Time constants and $\mathrm{S}_{1}$ lifetimes are reported in Table III.

Similar delay times $\tau_{\mathrm{d}}$ are obtained for all three TDDFT methods, indicating similar reaction pathways towards the $\operatorname{MECP}\left(\mathrm{C}_{2} \mathrm{C}_{3}\right)$ seam. However, the time constant $\tau_{\mathrm{e}}$ is $30 \%$ smaller at the SF-BH\&HLYP level as compared to the two LR-TDDFT methods, the latter of which are quite similar. This is likely due to the peaked topography near the $\operatorname{MECP}\left(\mathrm{C}_{2} \mathrm{C}_{3}\right)$ seam that is predicted by SF-BH\&HLYP, whereas the LR-TDDFT methods afford sloped CXs due to lack of proper coupling between $\mathrm{S}_{0}$ and $\mathrm{S}_{1}$ as discussed in Section IV B. Note that a peaked CX usually facilitates more efficient transitions than a sloped CX.

In Fig. 10, we compare the SF-BH\&HLYP and LR- $\omega$ B97X/TDA minimum-energy intersection seams around $\operatorname{MECP}\left(\mathrm{C}_{2} \mathrm{C}_{3}\right)$, by scanning the $\mathrm{C}_{2}=\mathrm{C}_{3}$ twist angle. The two seams are quite similar in shape except that the slope of the potential surface is larger in the LR- $\omega$ B97X/TDA case, with an energy change that is $\approx 3 \mathrm{kcal} / \mathrm{mol}$ greater over the same range of the torsion angle. The distribution of torsion angles around $\mathrm{C}_{2}=\mathrm{C}_{3}$ is plotted in Fig. 9(a) for the subset of activated trajectories that decay through $\operatorname{MECP}\left(\mathrm{C}_{2} \mathrm{C}_{3}\right)$, and the distribution around $\mathrm{C}_{1}-\mathrm{C}_{2}$ is plotted in Fig. 9(b). The similarities amongst these distributions provides further evidence that the trajectories produced by all three methods undergo the same reaction pathway. The fractions of cis photoproduct are 54\% (SF-BH\&HLYP), $53 \%$ (LR- $\omega$ B97X), and $47 \%$ (LR- $\omega$ B97X/TDA). The difference in $\tau_{\mathrm{e}}$ predicted by SF-BH\&HLYP and LR- $\omega$ B97X may therefore be only a consequence of different CX topographies. Nevertheless, these similarities are borne out only by removing dissimilar trajectories from the data set. Left to its own devices, LR-TDDFT (with or without the TDA) predicts rather different dynamics as compared to SF-BH\&HLYP.

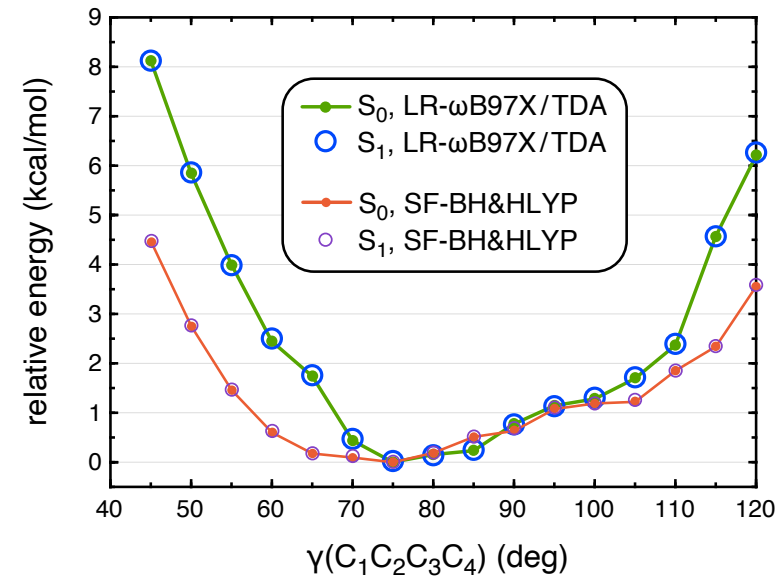

FIG. 10: Minimum-energy intersection seam between the $\mathrm{S}_{0}$ and $\mathrm{S}_{1}$ states of PSB3, obtained by scanning the twist angle of the $\mathrm{C}_{2}=\mathrm{C}_{3}$ double bond at two different levels of theory, as indicated.

\section{CONCLUSIONS}

We have carried out trajectory surface hopping simulations for the Schiff base model system $\mathrm{C}_{5} \mathrm{H}_{6} \mathrm{NH}_{2}^{+}$, in order to study the effects of correct versus incorrect $\mathrm{CX}$ topography on photodynamics. By comparing LR- and SF-TDDFT results, we observe that CXs predicted by the more traditional LR-TDDFT approach have sloped rather than peaked character, which is an artifact of improper couplings between ground (reference) and excited (response) states. The sloped character of the CX leads to a slowdown in the nonadiabatic dynamics and longer lifetimes in the $\mathrm{S}_{1}$ state, as compared to the SF-TDDFT simulations where the relevant CX is more strongly peaked. The SF-TDDFT results are generally in better agreement with existing CASPT2 estimates as compared to LR-TDDFT, although the short lifetime of the $\mathrm{S}_{1}$ state of $\mathrm{C}_{5} \mathrm{H}_{6} \mathrm{NH}_{2}^{+}$limits the magnitude of the discrepancies.

The main result of this work is a proof-of-concept demonstration that warped topography around a CX, resulting from an incorrect description of the topology of intersections involving $\mathrm{S}_{0}$ when LR-TDDFT is used, can manifest in photodynamics simulations if those simulations are carried all the way through to the final $\mathrm{S}_{1} \rightarrow \mathrm{S}_{0}$ deactivation event. Unless this problem is rectified, using SF-based versions of TDDFT or other methods, nonadiabatic simulations with LR-TDDFT should be halted prior to the point where trajectories return to the ground state. Information gained from the part of the trajectory that approaches the incorrectly-described CX is likely unreliable. 


\section{Acknowledgments}

This work was supported by the National Science Foundation (grant nos. CHE-1300603 and CHE-1665322) and calculations were performed at the Ohio Supercomputer Center (project no. PAA-0003). ${ }^{69}$ J.M.H. serves on the board of directors of Q-Chem Inc.

\section{Data availability statement}

The data that support the findings of this study are available from the corresponding author upon reasonable request.

\section{Appendix A: Nonadiabatic couplings between the reference and response states}

Using Eq. (2) for $\hat{H}$ and Eq. (11) for $\mathbf{h}_{I J}$, the nonadiabatic coupling between the HF ground state and a CIS excited state can be expressed as

$$
\begin{aligned}
\mathbf{h}_{0 I}^{\mathrm{CIS}}= & \left\langle\Phi_{0}\left|\left(\left|\Phi_{0}\right\rangle E_{0}\left\langle\Phi_{0}\right|\right)^{[\mathbf{R}]}\right| \Psi_{I}\right\rangle \\
& +\left\langle\Phi_{0}\left|\left(\sum_{i j a b}\left|\Phi_{i a}\right\rangle\left\langle\Phi_{i a}|\hat{H}| \Phi_{j b}\right\rangle\left\langle\Phi_{j b}\right|\right)^{[\mathbf{R}]}\right| \Psi_{I}\right\rangle \\
= & E_{0}\left\langle\Phi_{0}^{[\mathbf{R}]} \mid \Psi_{I}\right\rangle+E_{I} \sum_{i a}\left\langle\Phi_{0} \mid \Phi_{i a}^{[\mathbf{R}]}\right\rangle c_{i a}^{I} .
\end{aligned}
$$

By direct differentiation of the creation operators, ${ }^{34}$ it is possible to obtain the nuclear derivatives of the Slater determinants,

$$
\left|\Phi_{0}^{[\mathbf{R}]}\right\rangle=-\sum_{i a}\left\langle a^{[\mathbf{R}]} \mid i\right\rangle\left|\Phi_{i a}\right\rangle
$$

and

$$
\left\langle\Phi_{0} \mid \Phi_{i a}^{[\mathbf{R}]}\right\rangle=-\left\langle i^{[\mathbf{R}]} \mid a\right\rangle .
$$

Using the latter two results, Eq. (A1) can be simplified to afford the result that was given in Eq. (12).

The first-order derivative coupling vector is defined as

$$
\mathbf{d}_{I J}=\left\langle\Psi_{I} \mid \Psi_{J}^{[\mathbf{R}]}\right\rangle .
$$

The derivative coupling $\mathbf{d}_{0 I}^{\text {CIS }}$ between HF and CIS states can be derived by proceeding as above, and this affords

$$
\mathbf{d}_{0 I}^{\mathrm{CIS}}=-\sum_{i a}\left\langle a \mid i^{[\mathbf{R}]}\right\rangle c_{i a}^{I} .
$$

Comparing this expression to Eq. (12), one may conclude that the nonadiabatic coupling, as defined in Eq. (11), is related to the derivative coupling according to

$$
\mathbf{h}_{0 I}^{\mathrm{CIS}}=\left(E_{I}-E_{0}\right) \mathbf{d}_{0 I}^{\mathrm{CIS}} \text {. }
$$

Although Eq. (A6) is a standard expression in exact quantum mechanics, this relationship does not hold if the nonadiabatic coupling is defined using Eq. (13), for which $\mathbf{h}_{0 I}$ vanishes identically as a result of Brillouin's theorem.

Similarly, the nonadiabatic coupling between a groundstate Kohn-Sham determinant and a LR-TDDFT state may be defined as

$$
\mathbf{h}_{0 I}^{\mathrm{DFT}}=\omega_{I} \mathbf{d}_{0 I}^{\mathrm{DFT}}
$$

where $\omega_{I}=E_{I}-E_{0}$ is the LR-TDDFT excitation energy. The derivative coupling $\mathbf{d}_{0 I}^{\mathrm{DFT}}$ can be obtained using linear response theory. ${ }^{36}$ The result is

$$
\mathbf{d}_{0 I}^{\mathrm{DFT}}=-\sum_{i a}\left\langle a \mid i^{[\mathbf{R}]}\right\rangle\left(x_{i a}^{I}-y_{i a}^{I}\right) .
$$

The amplitudes $x_{i a}^{I}$ and $y_{i a}^{I}$ can be collected into vectors $\mathbf{x}^{I}$ and $\mathbf{y}^{I}$ that satisfy the LR-TDDFT eigenvalue equation, ${ }^{1-3}$

$$
\left(\begin{array}{ll}
\mathbf{A} & \mathbf{B} \\
\mathbf{B} & \mathbf{A}
\end{array}\right)\left(\begin{array}{l}
\mathbf{x}^{I} \\
\mathbf{y}^{I}
\end{array}\right)=\omega_{I}\left(\begin{array}{rr}
\mathbf{1} & \mathbf{0} \\
\mathbf{0} & -\mathbf{1}
\end{array}\right)\left(\begin{array}{l}
\mathbf{x}^{I} \\
\mathbf{y}^{I}
\end{array}\right) .
$$

The orbital Hessian matrices A and $\mathbf{B}$ have matrix elements

$$
\begin{aligned}
A_{a i, b j}= & \delta_{a b} \delta_{i j}\left(\varepsilon_{a}-\varepsilon_{i}\right)+(a i \mid j b) \\
& -C_{\mathrm{HF}}(a b \mid j i)+\left(1-C_{\mathrm{HF}}\right)\left(a i\left|\kappa_{\mathrm{xc}}\right| j b\right)
\end{aligned}
$$

and

$$
B_{a i, b j}=(a i \mid b j)-C_{\mathrm{HF}}(a j \mid b i)+\left(1-C_{\mathrm{HF}}\right)\left(a i\left|\kappa_{\mathrm{xc}}\right| b j\right),
$$

where $C_{\mathrm{HF}}$ indicates the fraction of exact exchange and $\kappa_{\mathrm{xc}}=\delta^{2} E_{\mathrm{xc}} / \delta \rho^{2}$ is the semilocal exchange-correlation kernel.

In order to compute $\mathbf{d}_{0 I}^{\mathrm{DFT}}$, the nuclear derivative of the MO coefficients needs to be evaluated:

$$
C_{\mu i}^{[\mathbf{R}]}=\sum_{a} C_{\mu a} U_{a i}^{[\mathbf{R}]}-\frac{1}{2} \sum_{j} C_{\mu j} S_{j i}^{[\overline{\mathbf{R}}]} .
$$

Here, the superscript $[\overline{\mathbf{R}}]$ represents a "skeleton" derivative, ${ }^{70}$ and $\mathbf{U}^{[\mathbf{R}]}$ satisfies the coupled-perturbed Kohn-Sham (CPKS) equation: ${ }^{71}$

$$
(\mathbf{A}+\mathbf{B}) \mathbf{U}^{[\mathbf{R}]}=-\mathbf{Q}^{[\overline{\mathbf{R}}]} .
$$

The right side of Eq. (A13) can be written as

$$
\begin{aligned}
Q_{a i}^{[\overline{\mathbf{R}}]}=F_{a i}^{[\overline{\mathbf{R}}]}-\varepsilon_{i} S_{a i}^{[\overline{\mathbf{R}}]} & \\
-\sum_{k l} & \left\{\left(1-C_{\mathrm{HF}}\right)\left(a i\left|\kappa_{\mathrm{xc}}\right| l k\right)+(a i \mid l k)\right. \\
& \left.-C_{\mathrm{HF}}[(a k \mid l i)+(a l \mid k i)]\right\} S_{k l}^{[\overline{\mathbf{R}}]}
\end{aligned}
$$

where $\mathbf{F}$ is the Fock matrix. 
Using Eq. (A13), the derivative coupling $\mathbf{d}_{0 I}^{\mathrm{DFT}}$ in Eq. (A8) can be recast as

$$
\mathbf{d}_{0 I}^{\mathrm{DFT}}=-\sum_{i a}\left(x_{i a}^{I}-y_{i a}^{I}\right)\left(U_{a i}^{[\mathbf{R}]}+\left\langle a \mid i^{[\overline{\mathbf{R}}]}\right\rangle\right) .
$$

From Eq. (A9), one may obtain

$$
(\mathbf{A}+\mathbf{B})\left(\mathbf{x}^{I}+\mathbf{y}^{I}\right)=\omega_{I}\left(\mathbf{x}^{I}-\mathbf{y}^{I}\right) .
$$

In conjunction with Eq. (A13), it is possible to show that $^{36}$

$$
-\left(\mathbf{x}^{I}-\mathbf{y}^{I}\right)^{\dagger} \mathbf{U}^{[\mathbf{R}]}=\omega_{I}^{-1}\left(\mathbf{x}^{I}+\mathbf{y}^{I}\right)^{\dagger} \mathbf{Q}^{[\overline{\mathbf{R}}]} .
$$

Substituting this expression into Eq. (A15), the derivative coupling $\mathbf{d}_{0 I}^{\mathrm{DFT}}$ is finally expressed $\mathrm{as}^{36}$

$$
\mathbf{d}_{0 I}^{\mathrm{DFT}}=\sum_{i a}\left[\omega_{I}^{-1}\left(x_{i a}^{I}+y_{i a}^{I}\right) Q_{i a}^{[\overline{\mathbf{R}}]}-\left(x_{i a}^{I}-y_{i a}^{I}\right)\left\langle a \mid i^{[\overline{\mathbf{R}}]}\right\rangle\right] .
$$

The nonadiabatic coupling defined in Eq. (A7) therefore takes the following form:

$$
\mathbf{h}_{0 I}^{\mathrm{DFT}}=\sum_{i a}\left[\left(x_{i a}^{I}+y_{i a}^{I}\right) Q_{i a}^{[\overline{\mathbf{R}}]}-\omega_{I}\left(x_{i a}^{I}-y_{i a}^{I}\right)\left\langle a \mid i^{[\overline{\mathbf{R}}]}\right\rangle\right] .
$$

Even if $\omega_{I}=0$, the first term on the right side of Eq. (A19) remains and does not appear to vanish. In the complete basis limit

$$
Q_{a i}^{[\overline{\mathbf{R}}]}=\left(a\left|v_{\mathrm{ne}}^{[\mathbf{R}]}\right| i\right),
$$

where $v_{\text {ne }}$ denotes the nucleus-electron Coulomb potential. Thus

$$
\mathbf{h}_{0 I}^{\mathrm{DFT}}=\operatorname{tr}\left[\left(\mathbf{x}^{I}+\mathbf{y}^{I}\right) \mathbf{v}_{\mathrm{ne}}^{[\overline{\mathbf{R}}]}\right] .
$$

Because the transition density matrix $\mathbf{x}^{I}+\mathbf{y}^{I}$ is generally nonzero even at a crossing point, the nonadiabatic coupling $\mathbf{h}_{0 I}^{\mathrm{DFT}}$ does not vanish either.

In the case of the CIS method, or if the TDA is applied to LR-TDDFT (by setting $y_{i a}^{I}=0$ ), then the derivative coupling becomes

$$
\mathbf{d}_{0 I}^{\mathrm{CIS} / \mathrm{TDA}}=-\sum_{i a} x_{i a}^{I}\left(U_{a i}^{[\mathbf{R}]}+\left\langle a \mid i^{[\overline{\mathbf{R}}]}\right\rangle\right) .
$$

Note that Eq. (A17) does not have a counterpart within the TDA, i.e.,

$$
-\left(\mathbf{x}^{I}\right)^{\dagger} \mathbf{U}^{[\mathbf{R}]} \neq \omega_{I}^{-1}\left(\mathbf{x}^{I}\right)^{\dagger} \mathbf{Q}^{[\overline{\mathbf{R}}]} .
$$

As such, the nonadiabatic coupling $\mathbf{h}_{0 I}^{\mathrm{TDA}}=\omega_{I} \mathbf{d}_{0 I}^{\mathrm{TDA}}$ vanishes when $\omega_{I}=0$.

\section{Appendix B: Topology of CXs between HF and CIS states}

In this section, we use a numerical example to demonstrate that the gradient difference vector $\mathbf{g} \equiv \mathbf{g}_{01}$ [Eq. (9)]

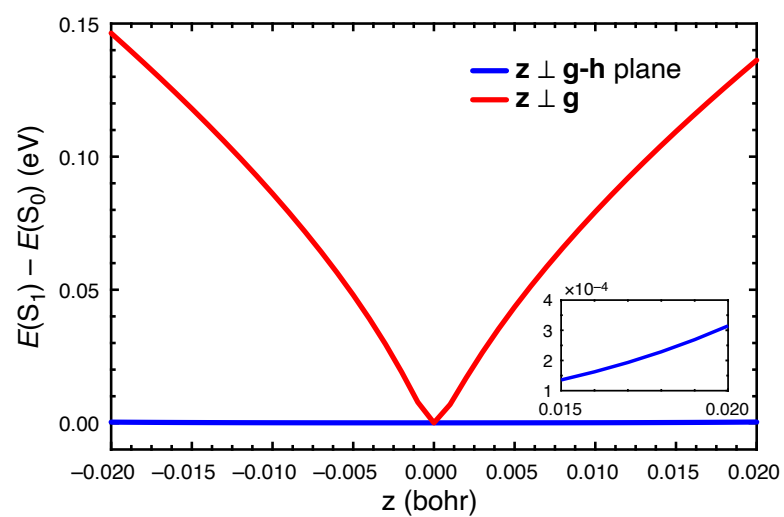

FIG. 11: Energy gaps between the $\mathrm{S}_{0}$ and $\mathrm{S}_{1}$ states of PSB3 computed at the CIS/6-31G* level. Two different director vectors $\mathbf{z}$ are used, corresponding to Eqs. (B1) and (B3) with the same choice of $\mathbf{R}$, and these are plotted in red and in blue, respectively. The value $\mathbf{z}=\mathbf{0}$ corresponds to the $\mathrm{S}_{0} / \mathrm{S}_{1}$ MECP geometry.

is not sufficient to determine the branching space at an approximate CX between the HF ground state and a CIS excited state. Consider an arbitrary nuclear geometry $\mathbf{R}$ and construct a unit vector $\mathbf{z}$ as follows:

$$
\mathbf{z}=\frac{\left(\mathbf{1}-\hat{\mathbf{g}} \hat{\mathbf{g}}^{\dagger}\right) \mathbf{R}}{\left\|\left(\mathbf{1}-\hat{\mathbf{g}} \hat{\mathbf{g}}^{\dagger}\right) \mathbf{R}\right\|}
$$

in which

$$
\hat{\mathrm{g}}=\mathbf{g} /\|\mathrm{g}\| \text {. }
$$

Thus, $\mathbf{z}$ is a normalized projection of the vector $\mathbf{R}$ onto the space orthogonal to $\mathbf{g}_{01}$. If the branching space were determined solely by the $\mathbf{g}$ vector, then we would expect that an intersection seam should exist along the direction of $\mathbf{z}$.

We have located the MECP along the conical seam between the $\mathrm{S}_{0}$ and $\mathrm{S}_{1}$ states of PSB3 at the CIS/6-31G* level of theory. The energy difference between the two states is less than $10^{-7}$ Ha. Taking this MECP as the origin, we carried out single-point energy scans for an arbitrary choice of $\mathbf{R}$, with $\mathbf{z}$ constructed from $\mathbf{R}$ according to Eq. (B1). The $\mathrm{S}_{1} / \mathrm{S}_{0}$ energy gap is plotted in Fig. 11 as the red curve. It can be seen that the degeneracy between the two states is lifted even in the very near vicinity of the MECP. This indicates that the $\mathbf{g}$ vector itself is not sufficient to determine the branching space.

As shown in Appendix $\mathrm{A}, \mathbf{h}_{0 I}^{\mathrm{CIS}}$ may be nonzero if $E_{I}-E_{0} \neq 0$, which implies that the $\mathbf{h}$ vector can also contribute to the branching space at an approximate CX. To determine whether this is numerically significant, we carried out the same energy scan described above (i.e., using the same vector $\mathbf{R}$ ), except that we make $\mathbf{z}$ perpendicular to both $\mathbf{g}$ and $\mathbf{h}$ :

$$
\mathbf{z}=\frac{\left(\mathbf{1}-\hat{\mathbf{g}} \hat{\mathbf{g}}^{\dagger}-\hat{\mathbf{y}} \hat{\mathbf{y}}^{\dagger}\right) \mathbf{R}}{\left\|\left(\mathbf{1}-\hat{\mathbf{g}} \hat{\mathbf{g}}^{\dagger}-\hat{\mathbf{y}} \hat{\mathbf{y}}^{\dagger}\right) \mathbf{R}\right\|}
$$


where

$$
\begin{aligned}
& \hat{\mathbf{y}}=\mathbf{y} /\|\mathbf{y}\| \\
& \mathbf{y}=\left(\mathbf{1}-\hat{\mathbf{g}} \hat{\mathbf{g}}^{\dagger}\right) \mathbf{h},
\end{aligned}
$$

with $\mathbf{h} \equiv \mathbf{h}_{01}^{\text {CIS }}$ defined in Eq. (12). The results are plotted as the blue curve in Fig. 11. This time, degeneracy between the $S_{0}$ and $S_{1}$ states is preserved along the $\mathbf{z}$ direction. Even at the distance of 0.02 bohr from the MECP geometry, the energy gap between the two states remains quite small $(\approx 0.0003 \mathrm{eV})$.

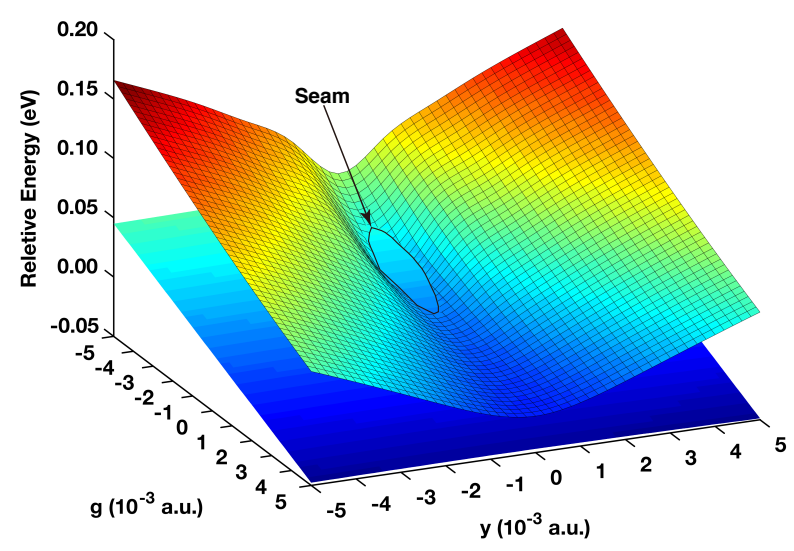

FIG. 12: Potential energy surfaces of the $\mathrm{S}_{0}$ and $\mathrm{S}_{1}$ states of PSB3 computed at CIS/6-31G* level of theory. Energies are measured relative to the $\mathrm{S}_{0}$ energy at the MECP geometry, which is taken as the coordinate origin. The intersection seam is highlighted in black.

Finally, starting from the MECP geometry we performed two-dimensional potential energy scans along the directions $\hat{\mathbf{g}}$ and $\hat{\mathbf{y}}$, with results plotted in Fig. 12. It can be seen that the intersection seam forms a closed curve encompassing a small area around the MECP, which is located at the origin. This behavior results from the fact that the $\mathrm{HF}$ reference state is unstable in the vicinity of the CX, leading to negative excitation energies. (In the absence of instabilities, one would expect a double-cone shape around the MECP.) Although these results are computed at the CIS level, one can expect the same behavior for intersections between the Kohn-Sham ground state and the LR-TDDFT excited states, at least within the pseudo-wave function formalism for DFT derivative couplings that is described herein.

When the excitation energy is small, we observe that the slope of the potential surfaces becomes unrealistically large near the seam, because the energy gradient has contributions that vary as $\omega_{I}^{-1} \cdot{ }^{72}$ This is clear by comparing Figs. 12 and 13, where the latter provides the analogous plot computed using SF-TDDFT around the same MECP. Here, a proper double cone is obtained. We conclude that the potential surfaces close to a crossing point involving the ground state generally exhibit incorrect topologies, at both the CIS level and at the LR-TDDFT level. This can be expected to have consequences in NMD simulations.

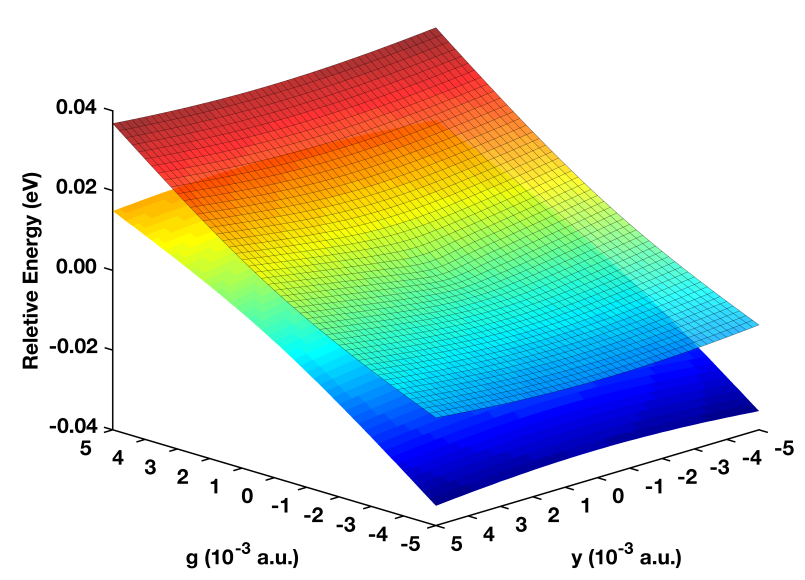

FIG. 13: Potential energy surfaces of the $S_{0}$ and $S_{1}$ states of PSB3 calculated at SF-BH\&HLYP/6-31G* level of theory. Energies are measured relative to the $\mathrm{S}_{0}$ energy at the MECP structure, which is taken as the coordinate origin. This plot exhibits the correct double-cone topology around the CX, whereas the analogous plot at the CIS level (Fig. 12) does not.
1 M. E. Casida, "Time-dependent density functional response theory for molecules", in Recent Advances in Density Functional Methods, Part I, D. P. Chong, Ed., Vol. I of Recent Advances in Computational Chemistry; World Scientific: River Edge, NJ, 1995; chapter 5, pages 155-192.

2 F. Furche, "On the density matrix based approach to timedependent density functional response theory", J. Chem. Phys., 114, 5982-5992 (2001).

3 A. Dreuw and M. Head-Gordon, "Single-reference ab initio methods for the calculation of excited states of large molecules", Chem. Rev., 105, 4009-4037 (2005).

4 B. G. Levine, C. Ko, J. Quenneville, and T. J. Martínez,
"Conical intersections and double excitations in timedependent density functional theory", Mol. Phys., 104, 1039-1051 (2006).

5 J. M. Herbert, X. Zhang, A. F. Morrison, and J. Liu, "Beyond time-dependent density functional theory using only single excitations: Methods for computational studies of excited states in complex systems", Acc. Chem. Res., 49, 931-941 (2016).

6 X. Zhang and J. M. Herbert, "Analytic derivative couplings for spin-flip configuration interaction singles and spin-flip time-dependent density functional theory", J. Chem. Phys., 141, 064104 (2014). 
7 Y. Shao, M. Head-Gordon, and A. I. Krylov, "The spin-flip approach within time-dependent density functional theory: Theory and applications to diradicals", J. Chem. Phys., 118, 4807-4818 (2003).

8 D. Casanova and A. I. Krylov, "Spin-flip methods in quantum chemistry", Phys. Chem. Chem. Phys., 22, 4326-4342 (2020).

9 Z. Li and W. Liu, "Spin-adapted open-shell random phase approximation and time-dependent density functional theory. I. Theory", J. Chem. Phys., 133, 064106 (2010).

10 Z. Li, W. Liu, Y. Zhang, and B. Suo, "Spin-adapted openshell time-dependent density functional theory. II. Theory and pilot application", J. Chem. Phys., 134, 134101 (2011).

11 Z. Li and W. Liu, "Spin-adapted open-shell timedependent density functional theory. III. An even better and simpler formulation", J. Chem. Phys., 135, 194106 (2011). Erratum: J. Chem. Phys., 138, 029904 (2013).

12 X. Zhang and J. M. Herbert, "Spin-flip, tensor equation-ofmotion configuration interaction with a density-functional correction: A spin-complete method for exploring excitedstate potential energy surfaces", J. Chem. Phys., 143, 234107 (2015).

13 S. Tretiak and S. Mukamel, "Density matrix analysis and simulation of electronic excitations in conjugated and aggregated molecules", Chem. Rev., 102, 3171-3212 (2002).

14 I. Tavernelli, U. F. Röhrig, and U. Rothlisberger, "Molecular dynamics in electronically excited states using timedependent density functional theory", Mol. Phys., 103, 963-981 (2005).

15 E. Tapavicza, I. Tavernelli, and U. Rothlisberger, "Trajectory surface hopping within linear response timedependent density functional theory", Phys. Rev. Lett., 98, 023001 (2007).

16 I. Tavernelli, E. Tapavicza, and U. Rothlisberger, "Nonadiabatic dynamics using time-dependent density functional theory: Assessing the coupling strengths", J. Mol. Struct. (Theochem), 914, 22-29 (2009).

17 B. F. E. Curchod, U. Rothlisberger, and I. Tavernelli, "Trajectory-based nonadiabatic dynamics with timedependent density functional theory", ChemPhysChem, 14, 1314-1340 (2013).

18 E. Tapavicza, G. D. Bellchambers, J. C. Vincent, and F. Furche, "Ab initio non-adiabatic molecular dynamics", Phys. Chem. Chem. Phys., 15, 18336-18348 (2013).

19 R. Crespo-Otero and M. Barbatti, "Recent advances and perspectives on nonadiabatic mixed quantum-classical dynamics", Chem. Rev., 118, 7026-7068 (2018).

${ }^{20}$ S. M. Parker, S. Roy, and F. Furche, "Multistate hybrid time-dependent density functional theory with surface hopping accurately captures ultrafast thymine photodeactivation", Phys. Chem. Chem. Phys., 21, 18999-19010 (2019).

21 L. Yue, Y. Liu, and C. Zhu, "Performance of TDDFT with and without spin-flip in trajectory surface hopping dynamics: cis-trans azobenzene photoisomerization", Phys. Chem. Chem. Phys., 20, 24123-24139 (2018).

22 M. Garavelli, P. Celani, F. Bernardi, M. A. Robb, and M. Olivucci, "The $\mathrm{C}_{5} \mathrm{H}_{6} \mathrm{NH}_{2}^{+}$protonated Shiff base: An ab initio minimal model for retinal photoisomerization", J. Am. Chem. Soc., 119, 6891-6901 (1997).

23 J. P. Malhado and J. T. Hynes, "Photoisomerization for a model protonated Schiff base in solution: Sloped/peaked conical intersection perspective", J. Chem. Phys., 137,
22A543 (2012).

24 S. Sekharan and K. Morokuma, "Why 11-cis-retinal? Why not 7-cis-, 9-cis-, or 13-cis-retinal in the eye?", J. Am. Chem. Soc., 133, 19052-19055 (2011).

25 S. Gozem, F. Melaccio, R. Lindh, A. I. Krylov, A. A. Granovsky, C. Angeli, and M. Olivucci, "Mapping the excited state potential energy surface of a retinal chromophore with multireference and equation-of-motion coupled-cluster methods", J. Chem. Theory Comput., 9, 4495-4506 (2013).

${ }^{26}$ Q. Ou, S. Fatehi, E. Alguire, Y. Shao, and J. E. Subotnik, "Derivative couplings between TD-DFT excited states obtained by direct differentiation in the Tamm-Dancoff approximation", J. Chem. Phys., 141, 024114 (2014).

27 Q. Ou, E. C. Alguire, and J. E. Subotnik, "Derivative couplings between time-dependent density functional theory excited states in the random-phase approximation based on pseudo-wavefunctions: Behavior around conical intersections", J. Phys. Chem. B, 119, 7150-7161 (2015).

28 Z. Li, B. Suo, and W. Liu, "First-order nonadiabatic coupling matrix elements between excited states: Implementation and application at the TD-DFT and pp-TDA levels", J. Chem. Phys., 141, 244105 (2014).

29 X. Zhang and J. M. Herbert, "Analytic derivative couplings in time-dependent density functional theory: Quadratic response theory versus pseudo-wavefunction approach", J. Chem. Phys., 142, 064109 (2015).

${ }^{30}$ Q. Ou, G. D. Bellchambers, F. Furche, and J. E. Subotnik, "First-order derivative couplings between excited states from adiabatic TDDFT response theory", J. Chem. Phys., 142, 064114 (2015).

31 S. M. Parker, D. Rappoport, and F. Furche, "Quadratic response properties from TDDFT: Trials and tribulations", J. Chem. Theory Comput., 14, 807-819 (2018).

32 D. R. Yarkony, "Diabolical conical intersections", Rev. Mod. Phys., 68, 985-1013 (1996).

33 S. Matsika and P. Krause, "Nonadiabatic events and conical intersections", Annu. Rev. Phys. Chem., 62, 621-643 (2011)

34 S. Fatehi, E. Alguire, Y. Shao, and J. E. Subotnik, "Analytic derivative couplings between configurationinteraction-singles states with built-in electron-transation factors for translational invariance", J. Chem. Phys., 135, 234105 (2011).

35 V. Chernyak and S. Mukamel, "Density-matrix representation of nonadiabatic couplings in time-dependent density functional (TDDFT) theories", J. Chem. Phys., 112, 3572-3579 (2000).

${ }^{36}$ R. Send and F. Furche, "First-order nonadiabatic couplings from time-dependent hybrid density functional response theory: Consistent formalism, implementation, and performance", J. Chem. Phys., 132, 044107 (2010).

37 E. C. Alguire, Q. Ou, and J. E. Subotnik, "Calculating derivative couplings between time-dependent HartreeFock excited states with pseudo-wavefunctions", J. Phys. Chem. B, 119, 7140-7149 (2015).

38 K. D. Closser, O. Gessner, and M. Head-Gordon, "Simulations of the dissociation of small helium clusters with $a b$ initio molecular dynamics in electronically excited states", J. Chem. Phys., 140, 134306 (2014).

39 Y. Harabuchi, K. Keipert, F. Zahariev, T. Taketsugu, and M. S. Gordon, "Dynamics simulations with spin-flip timedependent density functional theory: Photoisomerization and photocyclization mechanisms of cis-stilbene in $\pi \pi^{*}$ 
states", J. Phys. Chem. A, 118, 11987-11998 (2014).

40 N. Minezawa and M. S. Gordon, "Optimizing conical intersections by spin-flip density functional theory: Application to ethylene", J. Phys. Chem. A, 113, 12749-12753 (2009).

41 N. Minezawa and M. S. Gordon, "Photoisomerization of stilbene: A spin-flip density functional theory study", J. Phys. Chem. A, 115, 7901-7911 (2011).

42 Y. Harabuchi, S. Maeda, T. Taketsugu, N. Minezawa, and K. Morokuma, "Automated search for minimum energy conical intersection geometries between the lowest two singlet states $\mathrm{S}_{0} / \mathrm{S}_{1}$-MECIs by the spin-flip TDDFT method", J. Chem. Theory Comput., 9, 4116-4123 (2013).

43 N. Minezawa, "Optimizing minimum free-energy crossing points in solution: Linear-response free energy/spin-flip density functional theory approach", J. Chem. Phys., 141, 164118 (2014).

44 N. Minezawa and T. Nakajima, "Trajectory surface hopping molecular dynamics simulation by spin-flip timedependent density functional theory", J. Chem. Phys., 150, 204120 (2019).

45 E. Salazar and S. Faraji, "Theoretical study of cyclohexadiene/hexatriene photochemical interconversion using spinflip time-dependent density functional theory", Mol. Phys., 118, e1764120 (2020).

46 N. Minezawa and T. Nakajima, "Quantum mechanical/ molecular mechanical trajectory surface hopping molecular dynamics simulation by spin-flip time-dependent density functional theory", J. Chem. Phys., 152, 024119 (2020).

47 S. Lee, M. Filatov, S. Lee, and C. H. Choi, "Eliminating spin-contamination of spin-flip time dependent density functional theory within linear response formalism by the use of zeroth-order mixed-reference (MR) reduced density matrix", J. Chem. Phys., 149, 104101 (2018).

48 S. Lee, S. Shostak, M. Filatov, and C. H. Choi, "Conical intersections in organic molecules: Benchmarking mixedreference spin-flip time-dependent DFT (MSRF-TD-DFT) vs spin-flip TD-DFT", J. Phys. Chem. A, 123, 6455-6462 (2019).

49 S. Lee, E. E. Kim, H. Nakata, S. Lee, and C. H. Choi, "Efficient implementations of analytic energy gradient for mixed-reference spin-flip time-dependent density functional theory (MRSF-TDDFT)", J. Chem. Phys., 150, 184111 (2019).

50 Y. Horbatenko, S. Sadiq, S. Lee, M. Filatov, and C. H. Choi, "Mixed-reference spin-flip time-dependent density functional theory (MRSF-TDDFT) as a simply yet accurate method for diradicals and diradicaloids", J. Chem. Theory Comput., 17, 848-859 (2021).

51 Y. Horbatenko, S. Lee, M. Filatov, and C. H. Choi, "Performance analysis and optimization of mixed-reference spin-flip time-dependent density functional theory (MRSFTDDFT) for vertical excitation energies and singlet-triplet energy gaps", J. Phys. Chem. A, 123, 7991-8000 (2019).

52 B. R. Landry and J. E. Subotnik, "How to recover Marcus theory with fewest switches surface hopping: Add just a touch of decoherence", J. Chem. Phys., 137, 22A513 (2012).

53 A. Jain, E. Alguire, and J. E. Subotnik, "An efficient, augmented surface hopping algorithm that includes decoherence for use in large-scale simulations", J. Chem. Theory Comput., 12, 5256-5268 (2016).

54 J. E. Subotnik, A. Jain, B. Landry, A. Petit, W. Ouyang, and N. Bellonzi, "Understanding the surface hopping view of electronic transitions and decoherence", Annu. Rev.
Phys. Chem., 67, 387-417 (2016).

55 J. C. Tully, "Molecular dynamics with electronic transitions", J. Chem. Phys., 93, 1061-1071 (1990).

56 J.-D. Chai and M. Head-Gordon, "Systematic optimization of long-range corrected hybrid density functionals", J. Chem. Phys., 128, 084106 (2008).

57 A. D. Becke, "A new mixing of Hartree-Fock and local density-functional theories", J. Chem. Phys., 98, 13721377 (1993).

58 C. Lee, W. Yang, and R. G. Parr, "Development of the Colle-Salvetti correlation-energy formula into a functional of the electron density", Phys. Rev. B, 37, 785-789 (1988).

59 M. Huix-Rotllant, B. Natarajan, A. Ipatov, C. M. Wawire, T. Deutsch, and M. E. Casida, "Assessment of noncollinear spin-flip Tamm-Dancoff approximation timedependent density-functional theory for the photochemical ring-opening of oxirane", Phys. Chem. Chem. Phys., 12, 12811-12825 (2010).

${ }^{60} \mathrm{X}$. Zhang and J. M. Herbert, "Excited-state relaxation pathways in uracil versus hydrated uracil: Solvatochromatic shift in the ${ }^{1} n \pi^{*}$ state is the key", J. Phys. Chem. B, 118, 7806-7817 (2014).

61 Y. Shao, Z. Gan, E. Epifanovsky, A. T. B. Gilbert, M. Wormit, J. Kussmann, A. W. Lange, A. Behn, J. Deng, X. Feng, D. Ghosh, M. Goldey, P. R. Horn, L. D. Jacobson, I. Kaliman, R. Z. Khaliullin, T. Kús, A. Landau, J. Liu, E. I. Proynov, Y. M. Rhee, R. M. Richard, M. A. Rohrdanz, R. P. Steele, E. J. Sundstrom, H. L. Woodcock III, P. M. Zimmerman, D. Zuev, B. Albrecht, E. Alguire, B. Austin, G. J. O. Beran, Y. A. Bernard, E. Berquist, K. Brandhorst, K. B. Bravaya, S. T. Brown, D. Casanova, C.-M. Chang, Y. Chen, S. H. Chien, K. D. Closser, D. L. Crittenden, M. Diedenhofen, R. A. DiStasio Jr., H. Do, A. D. Dutoi, R. G. Edgar, S. Fatehi, L. Fusti-Molnar, A. Ghysels, A. Golubeva-Zadorozhnaya, J. Gomes, M. W. D. Hanson-Heine, P. H. P. Harbach, A. W. Hauser, E. G. Hohenstein, Z. C. Holden, T.-C. Jagau, H. Ji, B. Kaduk, K. Khistyaev, J. Kim, J. Kim, R. A. King, P. Klunzinger, D. Kosenkov, T. Kowalczyk, C. M. Krauter, K. U. Lao, A. Laurent, K. V. Lawler, S. V. Levchenko, C. Y. Lin, F. Liu, E. Livshits, R. C. Lochan, A. Luenser, P. Manohar, S. F. Manzer, S.-P. Mao, N. Mardirossian, A. V. Marenich, S. A. Maurer, N. J. Mayhall, C. M. Oana, R. Olivares-Amaya, D. P. O'Neill, J. A. Parkhill, T. M. Perrine, R. Peverati, P. A. Pieniazek, A. Prociuk, D. R. Rehn, E. Rosta, N. J. Russ, N. Sergueev, S. M. Sharada, S. Sharma, D. W. Small, A. Sodt, T. Stein, D. Stück, Y.-C. Su, A. J. W. Thom, T. Tsuchimochi, L. Vogt, O. Vydrov, T. Wang, M. A. Watson, J. Wenzel, A. White, C. F. Williams, V. Vanovschi, S. Yeganeh, S. R. Yost, Z.-Q. You, I. Y. Zhang, X. Zhang, Y. Zhao, B. R. Brooks, G. K. L. Chan, D. M. Chipman, C. J. Cramer, W. A. Goddard III, M. S. Gordon, W. J. Hehre, A. Klamt, H. F. Schaefer III, M. W. Schmidt, C. D. Sherrill, D. G. Truhlar, A. Warshel, X. Xu, A. Aspuru-Guzik, R. Baer, A. T. Bell, N. A. Besley, J.-D. Chai, A. Dreuw, B. D. Dunietz, T. R. Furlani, S. R. Gwaltney, C.-P. Hsu, Y. Jung, J. Kong, D. S. Lambrecht, W. Liang, C. Ochsenfeld, V. A. Rassolov, L. V. Slipchenko, J. E. Subotnik, T. Van Voorhis, J. M. Herbert, A. I. Krylov, P. M. W. Gill, and M. HeadGordon, "Advances in molecular quantum chemistry contained in the Q-Chem 4 program package", Mol. Phys., 113, 184-215 (2015)

62 O. Valsson, C. Filippi, and M. E. Casida, "Regarding 
the use and misuse of retinal protonated Schiff base photochemistry as a test case for time-dependent densityfunctional theory", J. Chem. Phys., 142, 144104 (2015).

63 M. A. Rohrdanz and J. M. Herbert, "Simultaneous benchmarking of ground- and excited-state properties with long-range-corrected density functional theory", J. Chem. Phys., 129, 034107 (2008).

${ }^{64}$ R. M. Richard and J. M. Herbert, "Time-dependent density-functional description of the ${ }^{1} \mathrm{~L}_{a}$ state in polycyclic aromatic hydrocarbons: Charge-transfer character in disguise?", J. Chem. Theory Comput., 7, 1296-1306 (2011).

65 F. Cordova, L. J. Doriol, A. Ipatov, M. E. Casida, C. Filippi, and A. Vela, "Troubleshooting time-dependent density-functional theory for photochemical applications: Oxirane", J. Chem. Phys., 127, 164111 (2007).

${ }^{66}$ L. Liu, J. Liu, and T. J. Martinez, "Dynamical correlation effects on photoisomerization: Ab initio multiple spawning dynamics with MS-CASPT2 for a model trans-protonated Schiff base", J. Phys. Chem. B, 120, 1940-1949 (2016).

67 D. R. Yarkony, "Nuclear dynamics near conical intersections in the adiabatic representation: I. The effects of lo- cal topography on interstate transitions", J. Chem. Phys., 114, 2601 (2001).

68 B. Efron and R. J. Tibshirani, An Introduction to the Bootstrap, Chapman \& Hall: New York, 1993.

69 "Ohio Supercomputer Center", http://osc.edu/ark:/19495/f5s1ph73.

70 Y. Yamaguchi, Y. Osamura, J. D. Goddard, and H. F. Schaefer III, A New Dimension to Quantum Chemistry: Analytic Derivative Methods in Ab Initio Molecular Electronic Structure Theory, Oxford University Press: New York, 1994.

${ }^{71}$ P. Deglmann, F. Furche, and R. Ahlrichs, "An efficient implementation of second analytical derivatives for density functional methods", Chem. Phys. Lett., 362, 511-518 (2002).

72 N. J. Russ, T. D. Crawford, and G. S. Tschumper, "Real versus artifactual symmetry-breaking effects in HartreeFock, density-functional, and coupled-cluster methods", J. Chem. Phys., 120, 7298-7306 (2004). 